\title{
A Single-Valued Extended Hesitant Fuzzy Score-Based Technique for Probabilistic Hesitant Fuzzy Multiple Criteria Decision-Making
}

\author{
Bahram Farhadinia (i) and Atefeh Taghavi \\ Department of Mathematics, Quchan University of Technology, Quchan, Iran \\ Correspondence should be addressed to Bahram Farhadinia; bfarhadinia@qiet.ac.ir
}

Received 19 June 2021; Revised 10 August 2021; Accepted 30 August 2021; Published 20 September 2021

Academic Editor: Ning Cai

Copyright ( 2021 Bahram Farhadinia and Atefeh Taghavi. This is an open access article distributed under the Creative Commons Attribution License, which permits unrestricted use, distribution, and reproduction in any medium, provided the original work is properly cited.

\begin{abstract}
The probabilistic hesitant fuzzy set (PHFS) is a worthwhile extension of the hesitant fuzzy set (HFS) which allows people to improve their quantitative assessment with the corresponding probability. Recently, in order to address the issue of difficulty in aggregating decision makers' opinions, a probability splitting algorithm has been developed that drives an efficient probabilisticunification process of PHFSs. Adopting such a unification process allows decision makers to disregard the probability part in developing fruitful theories of comparison of PHFSs. By keeping this feature in mind, we try to introduce a class of score functions for the notion of the single-valued extended hesitant fuzzy set (SVEHFS) as a novel deformation of PHFS. Interestingly, a SVEHFS not only belongs to a less dimensional space compared to that of PHFSs but also the proposed SVEHFS-based score functions satisfy a number of interesting properties. Eventually, some case studies of multiple criteria decision-making (MCDM) techniques under the PHFS environment are provided to demonstrate the effectiveness of proposed SVEHFS-based score functions.
\end{abstract}

\section{Introduction}

Hesitant fuzzy set (HFS) as an extension of the fuzzy set [1] was introduced for reflecting the hesitancy of decision makers in providing their preferences over alternatives such that the membership degree of an element in HFS is represented by a set of values in $[0,1]$. The concept of HFS is a field that still keeps attracting a significant amount of attention from researchers, and by owing to this concept, the other extensions of HFSs have been proposed in the literature [2-6] to overcome a number of corresponding challenges.

From diverse extensions of HFS, the concept of the extended hesitant fuzzy set (EHFS) is introduced first by Zhu and $\mathrm{Xu}$ [7] in terms of a function that returns a finite set of membership value-groups. Then, Farhadinia and HerreraViedma [8] re-visited and revised the notion of EHFS as the Cartesian product of " $n$ " HFSs in which each " $n$ "-tupleformed element of EHFS is referred to as the opinion of some decision makers simultaneously.
Another interesting generalization of HFSs occurs when we are required to provide experts' evaluations based on two cases: whether experts have the same weight or whether each value in a hesitant fuzzy element (HFE) gets the same probability distribution? These cases are covered by defining the concept of the probabilistic hesitant fuzzy set (PHFS) which was first developed by $\mathrm{Zhu}$ and $\mathrm{Xu}$ [9] to incorporate distribution information with the membership degrees included in hesitant fuzzy elements (HFEs). Furthermore, the PHFS concept has a great potential for handling multiple criteria decision-making (MCDM) processes in which both qualitative and quantitative criteria are to be considered [10-14].

Nowadays, among a large number of studies of PHFS notion, we may refer to the contribution of Zhang and $\mathrm{Wu}$ [15] in which two PHFS aggregation operators are developed by taking Archimedean $t$-norm and $t$-conorm into account. Following that work, $\mathrm{Li}$ and Wang [16] proposed the Hausdorff distance measure of PHFSs to extend a QUALItative FLEXible multiple (QUALIFLEX) technique for 
evaluating green suppliers. Yue et al. [17] developed the application of probabilistic hesitant fuzzy elements (PHFEs) in MCDM problems by proposing a set of probabilistic hesitant fuzzy aggregation operators. Following that, Zeng et al. [18] introduced the uncertain probabilistic-ordered weighted averaging distance operator in order to unify the framework between the probability and the ordered weighted averaging operator. Ding et al. [19] dealt with the situation in which the weight information is incomplete, and then, they concentrated on the class of PHFE-based multiple attribute group decision-making.

In a completely updated study, Farhadinia [20] pointed out that there exist two kinds of normalization processes in dealing with PHFS decision-making problems, namely, the probabilistic normalization and cardinal normalization. We need to mention that, among the contributions considering different types of probabilistic-unification processes, the most eminent works are those of Zhang et al. [21], Farhadinia and Xu [22], Farhadinia and Herrera-Viedma [23], Li and Wang [24], Wu et al. [25], and Lin et al. [26]. Except Lin et al.'s [26] probabilistic-unification process, Farhadinia [20] demonstrated that the other probabilistic-unification processes considered in the later-mentioned contributions are not reasonable from a mathematical point of view. It can be seen that the probabilistic-unification processes of Lin et al. [26] and Farhadinia [20] give rise to the same result with this difference that the process of Lin et al. compromises the unification of probabilities and HFE parts simultaneously, and that of Farhadinia unifies firstly the probabilities part, and then, it does the corresponding HFE part.

Keeping the latter-mentioned applications of PHFS notion in mind, the subject of PHFS ranking technique has received significant attention in the recent years. Up to now, a variety of PHFE comparison techniques have been proposed as the combination of hesitancy degree and its corresponding probability. Taking these two notions into account, there have been considerable contributions done in the past on the PHFE comparison techniques which were developed by employing the score and deviation values of each PHFE [14, 19, 21]. For instance, Lin et al. [26] put forward two types of probabilistic hesitant fuzzy aggregation operators for specifying the ranking results of alternatives in decision-making problems. Jiang and $\mathrm{Ma}$ [27] proposed a PHFE comparison technique using the arithmetic- and geometric-mean scores. Song et al. [28] presented a possibility degree formula for ranking PHFEs in the case where different PHFEs have common or intersecting values. This comparison technique is able to realize the optimal sorting under the hesitant fuzzy environment, and of course, it can reduce effectively the complexity of computation. Krishankumar et al. [29] suggested a ranking technique which extends a well-known VIKOR approach to the PHFS context. Wu et al. [30] supplied an enhanced satisfaction degree function on the basis of probabilistic hesitant fuzzy cumulative residual entropy for ranking the alternatives involved in a MCDM. Last but not least, Farhadinia and Xu [31] developed a thorough review of PHFS comparison techniques in MCDM and introduced a kind of PHFE ranking technique which is based on the multiplying and exponential deformation formulas of each element of a PHFE. They classified the PHFE measuring techniques in brief into the three classes which were called the elementbased processes for comparing PHFEs, the one step-based processes for comparing PHFEs, and the two step-based processes for comparing PHFEs.

However, the main objective of this study is to develop a class of score functions for capturing dependencies between PHFSs. Although the ranking of PHFSs has been discussed thoroughly before, the novelty presented here lies in the fact that the comparison is done inside the less dimensional space, referred here to as the single-valued EHFSs (SVEHFSs), and it has not yet been fully exploited. The notable characteristic of proposed SVEHFS-score functions is that not only they are projected from a highly dimensional space (i.e., the PHFS space) into a less dimensional space (i.e, the SVEHFS space) but also they offer a wide variety of interesting properties. Moreover, the proposed SVEHFS-based score functions proceed in less steps, and it relieves the laborious duty of using complex rules. Besides the latter advantages, we will demonstrate that the proposed SVEHFS-score functions can be more generalized to a wider class.

The organization of this contribution is as follows. We firstly review the process of unification of PHFSs in Section 2. Then, we demonstrate that how a unified PHFS is deduced to a SVEHFS in Section 3. Section 4 is devoted to introducing a new class of SVEHFS-based score functions for the unified PHFSs which provides the decision makers with more choices and flexibility. Subsequently, by re-encountering a number of MCDM problems, we indicate that the superiority of the proposed SVEHFS-score functions compare to the existing ones for PHFSs in Section 5. Section 6 concludes this contribution and provides some perspectives.

\section{The Probabilistic-Unification Process of PHFSs}

In the following part, we are going to review a number of basic notions which will be used frequently throughout this contribution.

By taking the reference set of $X$ into consideration, Torra [1] introduced the notion of hesitant fuzzy set (HFS) in terms of a function returning a finite subset of $[0,1]$ which is generally denoted by

$$
H=\{\langle x, h(x)\rangle: x \in X\},
$$

where $h(x) \in[0,1]$ is known as the hesitant fuzzy element (HFE) and denotes the possible membership degree of $x \in X$ to the set $H$.

There is another way of representing HFS already described in the form of

$$
H=\{\langle x, \underset{\hbar \in h(x)}{\cup}\{\hbar\}\rangle, x \in X\} .
$$

In order to emphasis on the probability occurrence of each possible value of HFE, Zhu [32] associated any element of HFE with its probability value as follows: 


$$
{ }^{\wp} H=\left\{\left\langle x,{ }^{\wp} h(x)\right\rangle: x \in X\right\}=\{\langle x, \underset{\langle\hbar(x), \mathfrak{Q}(x)\rangle \in \mathfrak{p} h(x)}{\cup}\{\langle\hbar(x), \wp(x)\rangle\}\rangle x \in X\}
$$

where ${ }^{\wp} h(x)$ stands for a probabilistic hesitant fuzzy element (PHFE).

As can be observed, any PHFE ${ }^{\wp} h(x)$ is a pair of possible membership degree $\hbar(x)$ and its probability distribution in the form of $\wp(x) \in[0,1]$ such that $\sum_{\mathfrak{s} h(x)}(\wp(x))=1$ for any $x \in X$.

It is obvious that if all the values of $\wp(x)$ are equal for any $x \in X$, then the PHFS ${ }^{r} H$ is reduced to a typical HFS.

Keeping the probabilistic-normalization and the cardinal-normalization procedures of PHFSs in mind, Farhadinia [20] represented a probabilistic-unification type of PHFSs. Anyway, it has been presented two PHFE probabilisticunification processes in earlier contributions, the one proposed by Farhadinia [20] and the other given by Lin et al. [26]. The main difference between these two processes is that Lin et al. [26] performed the unification process simultaneously for both the HFE part and its corresponding probability part, while Farhadinia [20] applied the unification process to probability part at the beginning and then partitioned the HFE part correspondingly. Regarding the same outcome of both Farhadinia's [20] and Lin et al.'s [26] procedures, we only consider the former one in the following.

By the use of Farhadinia's [20] algorithm which is seperated here as Algorithms 1 and 2, the initial partition of each PHFE probabilities is to be refined such that all the involved PHFEs have the same probability parts, while their corresponding HFE part remains unchanged. To explain Algorithm 1 and 2 briefly, we assume that ${ }^{8} h_{1}=U_{\left\langle\hbar_{1}, \wp_{1}\right\rangle \in \Re} h_{1}$ $\left\{\left\langle\hbar_{1}, \wp_{1}\right\rangle\right\}=\left\{\left\langle\hbar_{1}^{1}, \wp_{1}^{1}\right\rangle, \ldots,\left\langle\hbar_{1}^{l_{1}}, \wp_{1}^{l_{1}}\right\rangle\right\}, \quad{ }^{\natural} h_{2}=U_{\left\langle\hbar_{2}, \wp_{2}\right\rangle \in \mathfrak{r} h_{2}}$ $\left\{\left\langle\hbar_{2}, \wp_{2}\right\rangle\right\}=\left\{\left\langle\hbar_{2}^{1}, \wp_{2}^{1}\right\rangle, \ldots, \quad\left\langle\hbar_{2}^{l_{2}}, \quad \wp_{2}^{l_{2}}\right\rangle\right\}, \quad \ldots, \quad$ and ${ }^{\wp} h_{m}=\cup_{\left\langle\hbar_{m}, \wp_{m}\right\rangle \in \mathfrak{\wp}_{m}}\left\{\left\langle\hbar_{m}, \wp_{m}\right\rangle\right\}=\left\{\left\langle\hbar_{m}^{1}, \wp_{m}^{1}\right\rangle, \ldots,\left\langle\hbar_{m}^{l_{m}}, \wp_{m}^{l_{m}}\right\rangle\right\}$ indicate $m$ arbitrary PHFEs whose probabilities can be separated in the forms of $\left\{\wp_{1}^{1}, \wp_{1}^{2}, \ldots, \wp_{1}^{l_{1}}\right\}$ $\left\{\wp_{2}^{1}, \wp_{2}^{2}, \ldots, \wp_{2}^{l_{2}}\right\}, \ldots$, and $\left\{\wp_{m}^{1}, \wp_{m}^{2}, \ldots, \wp_{m}^{l_{m}}\right\}$, respectively. In this regard, the first phase of the PHFE probabilistic-unification process takes the following steps:

By setting $i:=i+1$, we return to Step 1 .

Farhadinia [20] indicated that the following results are the inherent advantages of the above-described algorithm.

Lemma 1 (see [20]). If $\prod^{*}:\left(\left\{\wp_{1}^{1}, \wp_{1}^{2}, \ldots, \wp_{1}^{l_{1}}\right\},\left\{\wp_{2}^{1}, \wp_{2}^{2}, \ldots\right.\right.$, $\left.\left.\wp_{2}^{l_{2}}\right\}, \ldots,\left\{\wp_{m}^{1}, \wp_{m}^{2}, \ldots, \wp_{m}^{l_{m}}\right\}\right) \longrightarrow\left\{\wp_{*}^{1}, \wp_{*}^{2}, \ldots, \wp_{*}^{l_{*}}\right\} \quad$ where $l_{*} \geq \max \left\{l_{1}, l_{2}, \ldots, l_{m}\right\}$, then the aggregation operator $\Pi^{*}$ is idempotent, commutative, and associative.

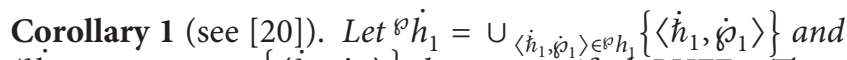
$\mathfrak{r} \dot{h}_{2}=U_{\left\langle\dot{h}_{2}, \dot{\beta}_{2}\right\rangle \in \mathfrak{p}_{2} h_{2}}\left\{\left\langle\dot{\hbar}_{2}, \dot{\boldsymbol{\wp}}_{2}\right\rangle\right\}$ be two unified PHFEs. Then, their corresponding probabilities sets are compatible (isomorphic).

To gain a more clear understanding of Farhadinia's [20] PHFE probabilistic-unification algorithm, we take the following three arbitrary PHFEs:

$$
\begin{aligned}
& { }^{\wp} h_{1}=\{\langle 0.3,0.2\rangle,\langle 0.6,0.5\rangle,\langle 0.8,0.3\rangle\}, \\
& { }^{8} h_{2}=\{\langle 0.4,0.5\rangle,\langle 0.7,0.5\rangle\}, \\
& { }^{8} h_{3}=\{\langle 0.2,0.1\rangle,\langle 0.5,0.7\rangle,\langle 0.9,0.2\rangle\} .
\end{aligned}
$$

It is apparent from illustrative Figures 1-3 that the unified forms are obtained in the forms of

$$
\begin{aligned}
& { }^{\mathfrak{}} \dot{h}_{1}=\{\langle 0.3,0.1\rangle,\langle 0.3,0.1\rangle,\langle 0.6,0.3\rangle,\langle 0.6,0.2\rangle,\langle 0.8,0.1\rangle,\langle 0.8,0.2\rangle\}, \\
& { }^{\wp} \dot{h}_{2}=\{\langle 0.4,0.1\rangle,\langle 0.4,0.1\rangle,\langle 0.4,0.3\rangle,\langle 0.7,0.2\rangle,\langle 0.7,0.1\rangle,\langle 0.7,0.2\rangle\}, \\
& { }^{\wp} \dot{h}_{3}=\{\langle 0.2,0.1\rangle,\langle 0.5,0.1\rangle,\langle 0.5,0.3\rangle,\langle 0.5,0.2\rangle,\langle 0.5,0.1\rangle,\langle 0.9,0.2\rangle\} .
\end{aligned}
$$

By the use of the above illustrative example, we find that the PHFE probabilistic-unification algorithm enables us to gain a set of PHFEs whose probabilities are in the form of a fixed vector.

\section{Reducing Unified PHFEs to SVEHFEs}

We can summarise the outcome of the previous section as follows: the PHFE probabilistic-unification algorithm leads to the set of HFE and probability pairs whose second part is a fixed vector.
As mentioned before, the purpose of this contribution is to propose a class of score functions for PHFSs with less involved factors. This fact would help us greatly reduce the model construction effort without losing the generality for different PHFSs; meanwhile, their probability part is common. Such an effort will result in defining a fundamental concept, called here as the single-valued extended hesitant fuzzy set (SVEHFS).

In the sequel, we shall present some preliminaries which will be useful for the establishment of the desired results. 
Initial step: consider the input probability sets as $\left\{\begin{array}{c}\left\{\wp_{1}^{1}, \wp_{1}^{2}, \ldots, \wp_{1}^{l_{1}}\right\} \\ \left\{\wp_{2}^{1}, \wp_{2}^{2}, \ldots, \wp_{2}^{l_{2}}\right\} \\ \vdots \\ \left\{\wp_{m}^{1}, \wp_{m}^{2}, \ldots, \wp_{m}^{l_{m}}\right\}\end{array}\right.$.

We now let $i=1$.

Step 1: compute $\wp_{*}^{i}=\min \left\{\wp_{1}^{i}, \wp_{2}^{i}, \ldots, \wp_{m}^{i}\right\}$.

Step 2: calculate the new probabilities: $\left\{\begin{array}{c}\wp_{1}^{i}:=\max \left\{\wp_{1}^{i}-\wp_{*}^{i}, 0\right\} ; \\ \wp_{2}^{i}:=\max \left\{\wp_{2}^{i}-\wp_{*}^{i}, 0\right\} ; \\ \vdots \\ \wp_{m}^{i}:=\max \left\{\wp_{m}^{i}-\wp_{*}^{i}, 0\right\} .\end{array}\right.$

Now, if $\wp_{1}^{i}=\wp_{2}^{i}=\cdots=\wp_{m}^{i}=0$, then STOP, and return $\wp_{*}=\left\{\wp_{*}^{1}, \wp_{*}^{2}, \ldots, \wp_{*}^{l_{*}}\right\}$ in which $l_{*} \geq \max \left\{l_{1}, l_{2}, \ldots, l_{m}\right\}$. Else, go to the

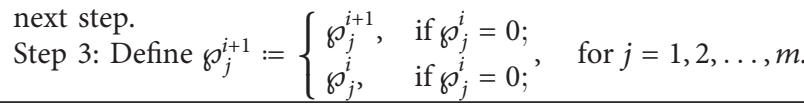

Algorithm 1: Phase 1 of Farhadinia's [20] algorithm.

Initial step: we assume that $\wp_{*}=\left\{\wp_{*}^{1}, \wp_{*}^{2}, \ldots, \wp_{*}^{l_{*}}\right\}$ is to be the output of Phase 1 of Farhadinia's [20] algorithm

$$
\begin{aligned}
& \wp_{1}^{1}=\sum_{k=1}^{k_{1}} \wp_{*}^{k}, \\
& \wp_{1}^{l_{1}}=\sum_{k=k_{l_{1}}+1}^{l_{1}} \wp_{*}^{k} . \\
& \left\langle\hbar_{1}^{1}, \wp_{*}^{1}\right\rangle, \ldots,\left\langle\hbar_{1}^{1}, \wp_{*}^{k_{1}}\right\rangle,
\end{aligned}
$$

Step 2: we re-arrange the HFE part of the first PHFE in the form of $\left\langle\hbar_{1}^{2}, \wp_{*}^{k_{1}+1}\right\rangle, \ldots,\left\langle\hbar_{1}^{2}, \wp_{*}^{k_{2}}\right\rangle$,

$$
\left\langle\hbar_{1}^{l_{1}}, \wp_{*}^{k_{l_{1}}+1}\right\rangle, \ldots,\left\langle\hbar_{1}^{l_{1}}, \wp_{*}^{k_{l^{*}}}\right\rangle .
$$

In summary, the unified form of the PHFE ${ }^{\wp} h_{1}$ will be ${ }^{\wp} \dot{h}_{1}=\left\{\left\langle\dot{\hbar}_{1}^{1}, \dot{\wp}_{1}^{1}\right\rangle, \ldots,\left\langle\dot{\hbar}_{1}^{k_{1}}, \dot{\wp}_{1}^{k_{1}}\right\rangle,\left\langle\dot{\hbar}_{1}^{k_{1}+1}, \dot{\wp}_{1}^{k_{1}+1}\right\rangle\right.$, $\ldots,\left\langle\dot{\hbar}_{1}^{k_{2}}, \dot{\wp}_{1}^{k_{2}}\right\rangle, \vdots\left\langle\dot{\hbar}_{1}^{k_{l_{1}+1}}, \dot{\wp}_{1}^{k_{1}+1}\right\rangle, \ldots,\left\langle\dot{\hbar}_{1}^{l^{*}}, \dot{\wp}_{1}^{l^{*}}\right\rangle$.

Step 3: in a similar manner as described above, we re-format the other PHFEs ${ }^{\wp} h_{2}, \ldots$, and ${ }^{\wp} h_{m}$ to ${ }^{\wp} \dot{h_{2}}, \ldots$, and ${ }^{\wp} \dot{h}_{m}$.

Algorithm 2: Phase 2 of Farhadinia's [20] algorithm.

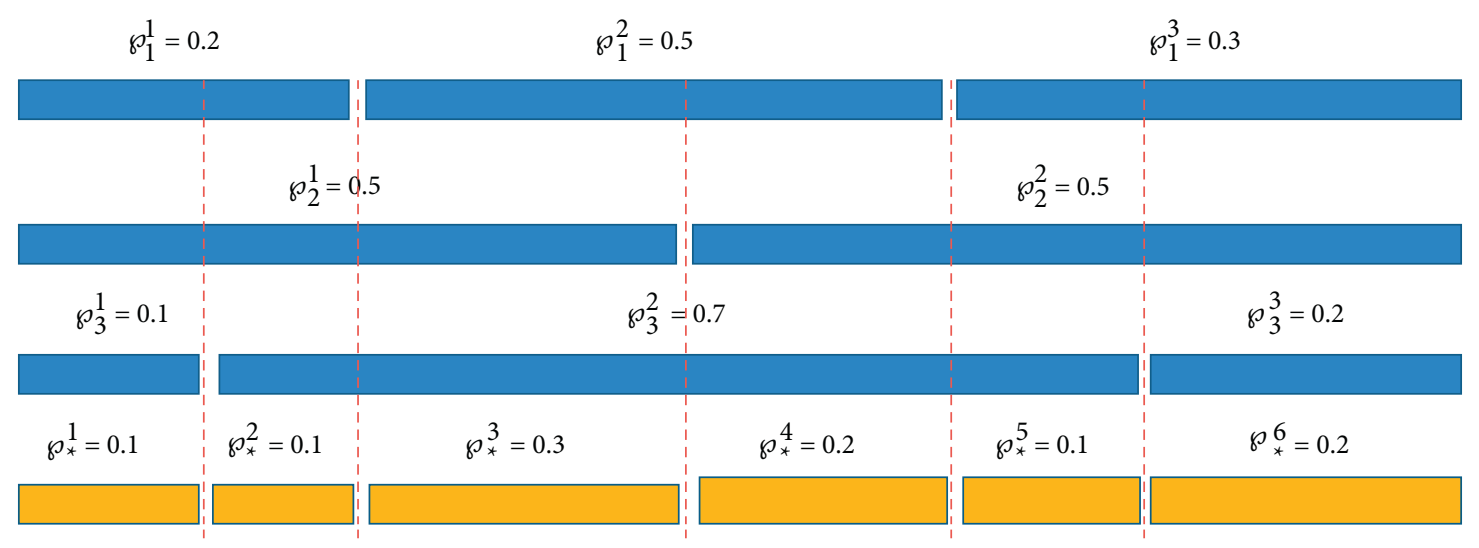

Figure 1: Stage 1 of the unification process. 


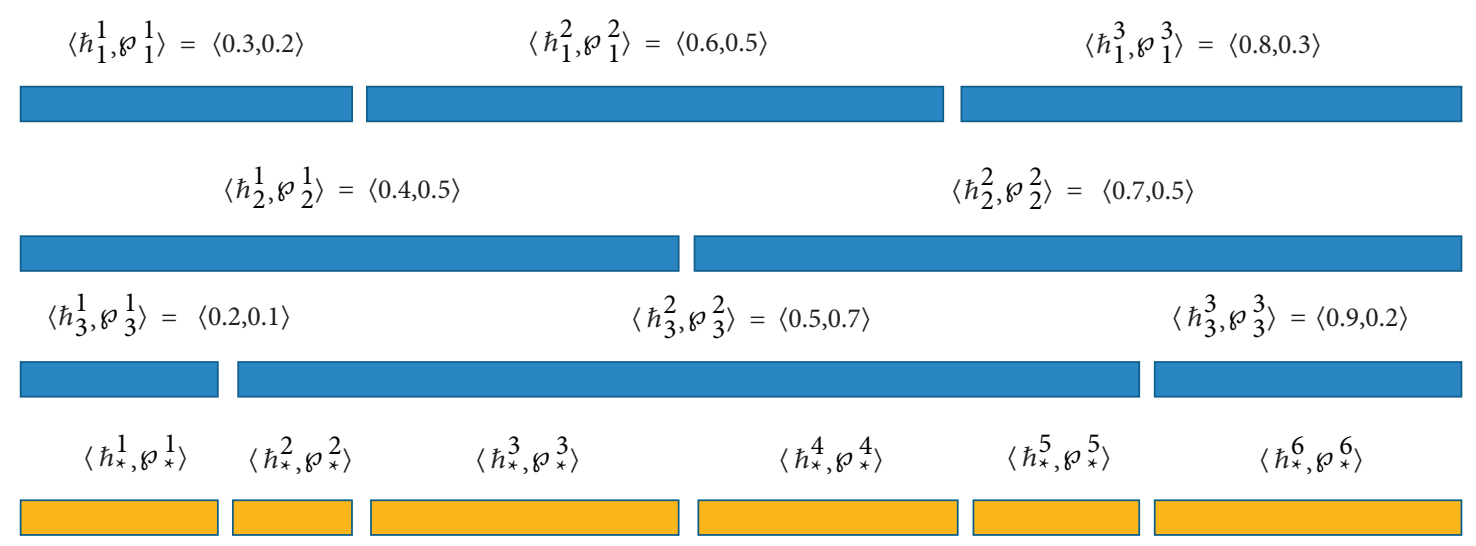

FIGURE 2: Stage 2 of the unification process.

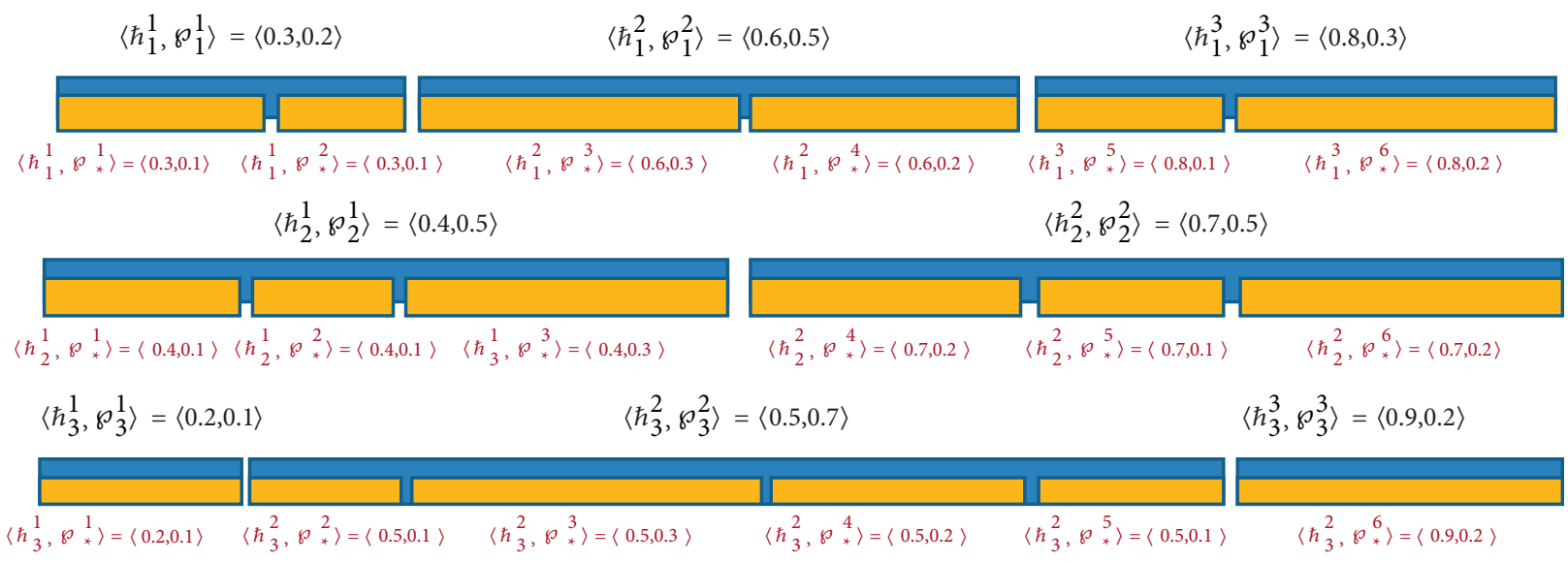

FIgure 3: Combining both Stages 1 and 2 of the unification process.

In a recent work, Zhu and $\mathrm{Xu}$ [7] introduced the notion of extended HFS (EHFS) in terms of a function which returns a finite set of membership value-groups. Then, Farhadinia and Herrera-Viedma [8] indicated that each element of an EHFS, known as the extended hesitant fuzzy element (EHFE), is indeed a set of $n$-tuples which demonstrates the opinion of $n$ number of decision makers. They introduced an extended hesitant fuzzy set (EHFS) on the reference set $X$ in the form of

$$
\mathbf{H}=\{\langle x, \mathbf{h}(x)\rangle \mid x \in X\}=\left\{\left\langle x, \underset{\left(\gamma_{1}(x), \ldots, \gamma_{m}(x)\right) \in \mathbf{h}(x)}{\cup}\left\{\left(\gamma_{1}(x), \ldots, \gamma_{m}(x)\right)\right\}\right\rangle \mid x \in X\right\},
$$

where

$$
\mathbf{h}=\underset{\left(\gamma_{1}, \ldots, \gamma_{m}\right) \in \mathbf{h}}{\cup}\left\{\left(\gamma_{1}, \ldots, \gamma_{m}\right)\right\}
$$

For instance, if we suppose that $X=\left\{x_{1}, x_{2}\right\}$ is the reference set and $\mathbf{h}_{1}(x)=\{(0.6,0.3,0.3),(0.5,0.2,0.2)\}$ and $\mathbf{h}_{2}(x)=\{(0.3,0.2,0.1)\}$ are two EHFEs on $X$, then the EHFS $\mathbf{H}$ is characterized by

which stands for an extended HFE (EHFE).

$$
\mathbf{H}=\left\{\left\langle x_{1}, \mathbf{h}_{1}(x)\right\rangle,\left\langle x_{2}, \mathbf{h}_{2}(x)\right\rangle\right\}=\left\{\left\langle x_{1},\{(0.6,0.3,0.3),(0.5,0.2,0.2)\}\right\rangle,\left\langle x_{2},\{(0.3,0.2,0.1)\}\right\rangle\right\} .
$$


Keeping the concept of EHFS in mind, we are now able to derive the concept of the single-valued extended hesitant fuzzy set (SVEHFS) as follows:

Definition 1. Let $\mathbf{H}=\left\{\left\langle x, \cup_{\left(\gamma_{1}(x), \ldots, \gamma_{m}(x)\right) \in \mathbf{h}(x)}\left\{\left(\gamma_{1}(x), \ldots\right.\right.\right.\right.$, $\left.\left.\left.\left.\gamma_{m}(x)\right)\right\}\right\rangle \mid x \in X\right\}$ be an extended hesitant fuzzy set (EHFS) on the reference set $X$. A single-valued extended hesitant fuzzy set (SVEHFS) is interpreted as the reduced form of $\mathbf{H}$ being characterized by

$$
\dot{H}=\left\{\left\langle x,\left\{\left(\gamma_{1}(x), \ldots, \gamma_{m}(x)\right)\right\}\right\rangle \mid x \in X\right\},
$$

where, for a fixed $x \in X$,

$$
\mathbf{h}(x)=\left\{\left(\gamma_{1}(x), \ldots, \gamma_{m}(x)\right)\right\},
$$

which stands for a single-valued extended hesitant fuzzy element (SVEHFE).

To give a more specific example, let us consider again the above example of EHFS, but in the form of SVEHFS, suppose that $X=\left\{x_{1}, x_{2}\right\}$ is the reference set and $\mathbf{h}_{1}(x)=$ $\{(0.6,0.3,0.3)\}$ and $\mathbf{h}_{2}(x)=\{(0.3,0.2,0.1)\}$ are two SVEHFEs on $X$. Then, the SVEHFS $\dot{H}$ is characterized by

$$
\dot{H}=\left\{\left\langle x_{1}, \mathbf{h}_{1}(x)\right\rangle,\left\langle x_{2}, \mathbf{h}_{2}(x)\right\rangle\right\}=\left\{\left\langle x_{1},\{(0.6,0.3,0.3)\}\right\rangle,\left\langle x_{2},\{(0.3,0.2,0.1)\}\right\rangle\right\} .
$$

Now, we turn back to the beginning expression in this section where it was stated that all of the pairs involved in a unified PHFE correspond to a fixed vector as their probability part.

If we put aside the probability part of the unified PHFEs, then it gives rise to forming the corresponding SVEHFEs.
For more explanation, we suppose that ${ }^{\wp} h_{1}=$ $\left\langle\hbar_{1}^{1}, \wp_{1}^{1}\right\rangle,\left\langle\hbar_{1}^{2}, \wp_{1}^{2}\right\rangle,\left\langle\hbar_{1}^{3}, \wp_{1}^{3}\right\rangle, \wp_{2} h_{2}=\left\{\left\langle\hbar_{2}^{1}, \wp_{2}^{1}\right\rangle,\left\langle\hbar_{2}^{2}, \wp_{2}^{2}\right\rangle\right\}$, and $\wp h_{3}=\left\{\left\langle\hbar_{3}^{1}, \wp_{3}^{1}\right\rangle,\left\langle\hbar_{3}^{2}, \wp_{3}^{2}\right\rangle,\left\langle\hbar_{3}^{3}, \wp_{3}^{3}\right\rangle\right\}$ are three arbitrary PHFEs. Then, their unified forms can be derived as follows:

$$
\begin{aligned}
& { }^{\wp} \dot{h}_{1}=\left\{\left\langle\hbar_{1}^{1}, \wp_{*}^{1}\right\rangle,\left\langle\hbar_{1}^{1}, \wp_{*}^{2}\right\rangle,\left\langle\hbar_{1}^{2}, \wp_{*}^{3}\right\rangle,\left\langle\hbar_{1}^{2}, \wp_{*}^{4}\right\rangle,\left\langle\hbar_{1}^{3}, \wp_{*}^{5}\right\rangle,\left\langle\hbar_{1}^{3}, \wp_{*}^{6}\right\rangle\right\}, \\
& { }^{\wp} \dot{h}_{2}=\left\{\left\langle\hbar_{2}^{1}, \wp_{*}^{1}\right\rangle,\left\langle\hbar_{2}^{1}, \wp_{*}^{2}\right\rangle,\left\langle\hbar_{2}^{1}, \wp_{*}^{3}\right\rangle,\left\langle\hbar_{2}^{2}, \wp_{*}^{4}\right\rangle,\left\langle\hbar_{2}^{2}, \wp_{*}^{5}\right\rangle,\left\langle\hbar_{2}^{2}, \wp_{*}^{6}\right\rangle\right\}, \\
& { }^{\wp} \dot{h}_{3}=\left\{\left\langle\hbar_{3}^{1}, \wp_{*}^{1}\right\rangle,\left\langle\hbar_{3}^{2}, \wp_{*}^{2}\right\rangle,\left\langle\hbar_{3}^{2}, \wp_{*}^{3}\right\rangle,\left\langle\hbar_{3}^{2}, \wp_{*}^{4}\right\rangle,\left\langle\hbar_{3}^{2}, \wp_{*}^{5}\right\rangle,\left\langle\hbar_{3}^{3}, \wp_{*}^{6}\right\rangle\right\} .
\end{aligned}
$$

If we put all the same probability part $\left(\wp_{*}^{1}, \wp_{*}^{2}, \ldots, \wp_{*}^{6}\right)$ of the later unified PHFEs $\mathscr{\wp} \dot{h}_{1}, \mathfrak{\wp} \dot{h}_{2}$, and $\mathscr{\wp} h_{3}$ aside, then the corresponding SVEHFEs are given as follows:

$$
\begin{aligned}
& \dot{h}_{1}:=\mathbf{h}_{1}=\left\{\left(\hbar_{1}^{1}, \hbar_{1}^{1}, \hbar_{1}^{2}, \hbar_{1}^{2}, \hbar_{1}^{3}, \hbar_{1}^{3}\right)\right\}, \\
& \dot{h}_{2}:=\mathbf{h}_{2}=\left\{\left(\hbar_{2}^{1}, \hbar_{2}^{1}, \hbar_{2}^{1}, \hbar_{2}^{2}, \hbar_{2}^{2}, \hbar_{2}^{2}\right)\right\}, \\
& \dot{h}_{3}:=\mathbf{h}_{3}=\left\{\left(\hbar_{3}^{1}, \hbar_{3}^{2}, \hbar_{3}^{2}, \hbar_{3}^{2}, \hbar_{3}^{2}, \hbar_{3}^{3}\right)\right\} .
\end{aligned}
$$

Before ending this section, we are required to discuss about the issue of distance measures for SVEHFEs. Generally, an unified-PHFE distance measure is constructed using the different part of hesitancy and probability parts. This is while the probability part of PHFEs is released in defining the concept of SVEHFEs. Therefore, the probability difference part may not make sense in developing distance measures for SVEHFEs, and only the hesitancy difference part is kept instead.

Now, if we assume that the weight of element $x_{i} \in X$ is to be denoted by $w_{i}$, satisfying $w_{i} \in[0,1]$ and $\sum_{i=1}^{N} w_{i}=1$, then a series of weighted distance measures for SVEHFSs $\dot{H}_{1}=$ $\left\{\left\langle x, \dot{h}_{1}(x)=\left\{\left(\gamma_{1}^{1}(x), \ldots, \gamma_{m}^{1}(x)\right)\right\}\right\rangle: x \in X\right\}$ and $\dot{H}_{2}=\{\langle x$, $\left.\left.\dot{h}_{2}(x)=\left\{\left(\gamma_{1}^{2}(x), \ldots, \gamma_{m}^{2}(x)\right)\right\}\right\rangle: x \in X\right\}$ will be developed as the following:

(1) The single-valued extended hesitant weighted distance measure:

$$
\mathrm{d}_{1}\left(\dot{H}_{1}, \dot{H}_{2}\right)=\left[\sum_{i=1}^{N} w_{i}\left(\frac{1}{m} \sum_{j=1}^{m}\left|\gamma_{j}^{1}\left(x_{i}\right)-\gamma_{j}^{2}\left(x_{i}\right)^{\lambda}\right|\right)\right]^{1 / \lambda}, \quad \lambda>0 .
$$

(2) The single-valued extended hesitant weighted Hausdorff distance measure:

$$
\mathrm{d}_{2}\left(\dot{H}_{1}, \dot{H}_{2}\right)=\left[\sum_{i=1}^{N} w_{i} \max _{1 \leq j \leq m}\left\{\left|\gamma_{j}^{1}\left(x_{i}\right)-\gamma_{j}^{2}\left(x_{i}\right)\right|^{\lambda}\right\}\right]^{1 / \lambda}, \quad \lambda>0 .
$$

(3) The single-valued extended hesitant weighted hybrid distance measure:

$$
\mathrm{d}_{3}\left(\dot{H}_{1}, \dot{H}_{2}\right)=\left[\sum_{i=1}^{N} w_{i} \times \frac{1}{2}\left(\frac{1}{m} \sum_{j=1}^{m}\left|\gamma_{j}^{1}\left(x_{i}\right)-\gamma_{j}^{2}\left(x_{i}\right)\right|^{\lambda}+\max _{1 \leq j \leq m}\left\{\left|\gamma_{j}^{1}\left(x_{i}\right)-\gamma_{j}^{2}\left(x_{i}\right)\right|\right\}\right)\right]^{1 / \lambda}, \quad \lambda>0 .
$$


(4) The generalized single-valued extended hesitant weighted hybrid distance measure:

$$
\mathrm{d}_{g}\left(\dot{H}_{1}, \dot{H}_{2}\right)=\left[\sum_{i=1}^{N} w_{i} \times \alpha\left(\frac{1}{m} \sum_{j=1}^{m}\left|\gamma_{j}^{1}\left(x_{i}\right)-\gamma_{j}^{2}\left(x_{i}\right)\right|^{\lambda}\right)+\beta\left(\max _{1 \leq j \leq m}\left\{\left|\gamma_{j}^{1}\left(x_{i}\right)-\gamma_{j}^{2}\left(x_{i}\right)\right|^{\lambda}\right\}\right)\right]^{1 / \lambda}, \quad \lambda>0,
$$

where $0 \leq \alpha, \beta \leq 1$, and $\alpha+\beta=1$.

Needless to say that all the abovementioned distance measures $d_{1}(.,),. d_{2}(.,$.$) , and d_{3}(.,$.$) can be derived from the$ generalized form of $d_{g}(.,$.$) .$

Theorem 1. Let $\dot{H}_{1}=\left\{\left\langle x, \dot{h}_{1}(x)=\left\{\left(\gamma_{1}^{1}(x), \ldots, \quad \gamma_{m}^{1}\right.\right.\right.\right.$ $(x))\}\rangle: x \in X\} \quad$ and $\quad \dot{H}_{2}=\left\{\left\langle x, \dot{h}_{2}(x)=\left\{\left(\gamma_{1}^{2}(x), \ldots\right.\right.\right.\right.$, $\left.\left.\left.\left.\gamma_{m}^{2}(x)\right)\right\}\right\rangle: x \in X\right\}$ be two SVEHFSs. Then, the weighted distance measures for SVEHFSs given by (21)-(24) satisfy the following properties:

(1) $0 \leq d\left(\dot{H}_{1}, \dot{H}_{2}\right) \leq 1$

(2) $d\left(\dot{H}_{1}, \dot{H}_{2}\right)=0$ if and only if $\dot{H}_{1}=\dot{H}_{2}$

(3) $d\left(\dot{H}_{1}, \dot{H}_{2}\right)=d\left(\dot{H}_{2}, \dot{H}_{1}\right)$
(4) $d\left(\dot{H}_{1}, \dot{H}_{2}\right) \leq d\left(\dot{H}_{1}, \dot{H}_{3}\right)$ if $\dot{H}_{1} \leq{ }_{H} \dot{H}_{2} \leq{ }_{H} \dot{H}_{3}$ implying that $\dot{h}_{1}(x) \leq_{h} \dot{h}_{2}(x) \leq_{h} \dot{h}_{3}(x)$ for any $x \in X$, that is, $\gamma_{j}^{1}(x) \leq \gamma_{j}^{2}(x) \leq \gamma_{j}^{3}(x)$ for any $j=1,2, \ldots, m$

Proof. We only prove the above assertions for the distance measure $\mathrm{d}_{g}(.,$.$) given by (17), and the others can be deduced$ easily.

Axiom 1. Keeping equation (17) in mind, we easily deduce that $0 \leq\left|\gamma_{j}^{1}\left(x_{i}\right)-\gamma_{j}^{2}\left(x_{i}\right)\right| \leq 1$ for any $0 \leq \gamma_{j}^{1}\left(x_{i}\right) \leq 1$ and $0 \leq \gamma_{j}^{2}\left(x_{i}\right) \leq 1$ in which $i=1,2, \ldots, N$ and $j=1,2, \ldots, m$. These easily give rise to

$$
0 \leq \frac{1}{m} \sum_{j=1}^{m}\left|\gamma_{j}^{1}\left(x_{i}\right)-\gamma_{j}^{2}\left(x_{i}\right)\right|^{\lambda} \leq 1, \quad \text { and } 0 \leq \max _{1 \leq j \leq m}\left\{\left|\gamma_{j}^{1}\left(x_{i}\right)-\gamma_{j}^{2}\left(x_{i}\right)\right|^{\lambda}\right\} \leq 1,
$$

for any $\lambda>0$. Now, by taking $w_{i} \in[0,1]$ and $\sum_{i=1}^{N} w_{i}=1$, and Axiom 2. Let moreover, $0 \leq \alpha, \beta \leq 1$ and $\alpha+\beta=1$, we result in $0 \leq \mathrm{d}_{g}\left(\dot{H}_{1}, \dot{H}_{2}\right) \leq 1$.

$$
\mathrm{d}_{g}\left(\dot{H}_{1}, \dot{H}_{2}\right)=\left[\sum_{i=1}^{N} w_{i} \times \alpha\left(\frac{1}{m} \sum_{j=1}^{m}\left|\gamma_{j}^{1}\left(x_{i}\right)-\gamma_{j}^{2}\left(x_{i}\right)\right|^{\lambda}\right)+\beta\left(\max _{1 \leq j \leq m}\left\{\left|\gamma_{j}^{1}\left(x_{i}\right)-\gamma_{j}^{2}\left(x_{i}\right)\right|^{\lambda}\right\}\right)\right]^{1 / \lambda}=0 .
$$

This implies that

$$
\frac{1}{m} \sum_{j=1}^{m}\left|\gamma_{j}^{1}\left(x_{i}\right)-\gamma_{j}^{2}\left(x_{i}\right)\right|^{\lambda}=0, \quad \text { and } \max _{1 \leq j \leq m}\left\{\left|\gamma_{j}^{1}\left(x_{i}\right)-\gamma_{j}^{2}\left(x_{i}\right)\right|^{\lambda}\right\}=0 \text {, }
$$

in which both of them lead to $\left|\gamma_{j}^{1}\left(x_{i}\right)-\gamma_{j}^{2}\left(x_{i}\right)\right|=0$, that is, $\gamma_{j}^{1}\left(x_{i}\right)=\gamma_{j}^{2}\left(x_{i}\right)$ for any $i=1,2, \ldots, N$ and $j=1,2, \ldots, m$. Thus, we conclude that $\dot{H}_{1}=\dot{H}_{2}$.

The inverse axiom will be easily proved in the same manner.
Axiom 3. The proof is immediate from definition of distance measure $\mathrm{d}_{g}(.,$.$) given by (17).$

Axiom 4. If $\dot{H}_{1} \leq{ }_{H} \dot{H}_{2} \leq{ }_{H} \dot{H}_{3}$, then it implies that $\dot{h}_{1}\left(x_{i}\right) \leq{ }_{h} \dot{h}_{2}\left(x_{i}\right) \leq{ }_{h} \dot{h}_{3}\left(x_{i}\right)$ for any $x_{i} \in X$, that is, 
$\gamma_{j}^{1}\left(x_{i}\right) \leq \gamma_{j}^{2}\left(x_{i}\right) \leq \gamma_{j}^{3}\left(x_{i}\right)$ for any $j=(1,2, \ldots, m)$ and $x_{i} \in X$. The latter inequalities give rise to $\left|\gamma_{j}^{1}\left(x_{i}\right)-\gamma_{j}^{2}\left(x_{i}\right)\right| \leq$ $\left|\gamma_{j}^{1}\left(x_{i}\right)-\gamma_{j}^{3}\left(x_{i}\right)\right|$ for any $k=(1,2, \ldots, m)$ and $x_{i} \in X$, and therefore,

$$
\begin{aligned}
\mathrm{d}_{g}\left(\dot{H}_{1}, \dot{H}_{2}\right) & =\left[\sum_{i=1}^{N} w_{i} \times \alpha\left(\frac{1}{m} \sum_{j=1}^{m}\left|\gamma_{j}^{1}\left(x_{i}\right)-\gamma_{j}^{2}\left(x_{i}\right)\right|^{\lambda}\right)+\beta\left(\max _{1 \leq j \leq m}\left\{\left|\gamma_{j}^{1}\left(x_{i}\right)-\gamma_{j}^{2}\left(x_{i}\right)\right|^{\lambda}\right\}\right)\right]^{1 / \lambda} \\
& \leq\left[\sum_{i=1}^{N} w_{i} \times \alpha\left(\frac{1}{m} \sum_{j=1}^{m}\left|\gamma_{j}^{1}\left(x_{i}\right)-\gamma_{j}^{3}\left(x_{i}\right)\right|^{\lambda}\right)+\beta\left(\max _{1 \leq j \leq m}\left\{\left|\gamma_{j}^{1}\left(x_{i}\right)-\gamma_{j}^{3}\left(x_{i}\right)\right|^{\lambda}\right\}\right)\right]^{1 / \lambda}=\mathrm{d}_{g}\left(\dot{H}_{1}, \dot{H}_{3}\right) .
\end{aligned}
$$

\section{SVEHFS-Based Score Function for PHFSs}

As will be shown later, the score function of SVEHFS is fundamentally defined in accordance with the score function of its SVEHFEs, and therefore, we only discuss the score functions for SVEHFEs.

Now, we are in a position to introduce a class of SVEHFE-score functions by the help of SVEHFE distance measures given by (14)-(17).

Definition 2. Let $\dot{h}=\left\{\left(\gamma_{1}, \ldots, \gamma_{m}\right)\right\}$ be a SVEHFE. The score function $S c($.$) is defined as$

$$
S c(\dot{h}):=S c\left(\left(\gamma_{1}, \ldots, \gamma_{m}\right)\right)=1-\mathrm{d}\left(\left(\gamma_{1}, \ldots, \gamma_{m}\right), 1\right),
$$

where $\mathrm{d}(.,$.$) is a distant measure for SVEHFE and 1$ stands for the $\operatorname{SVEHFE}(1, \ldots, 1)$.
As will be shown below, score function (22) satisfies the fundamental properties, known as monotonicity, boundary conditions, idempotency, and duality.

Property 1 (monotonicity). Let $\dot{h}_{1}=\left\{\left(\gamma_{1}^{1}, \ldots, \gamma_{m}^{1}\right)\right\}$ and $\dot{h}_{2}=$ $\left\{\left(\gamma_{1}^{2}, \ldots, \gamma_{m}^{2}\right)\right\}$ be two SVEHFEs such that $\dot{h}_{1} \leq_{h} \dot{h}_{2}$, that is, $\gamma_{j}^{1} \leq \gamma_{j}^{2}$ for any $j=1,2, \ldots, m$. Then, the score function $\operatorname{Sc}($.) given by (22) satisfies

$$
\operatorname{Sc}\left(\dot{h}_{1}\right) \leq \operatorname{Sc}\left(\dot{h}_{2}\right)
$$

Proof. From the fact that $\dot{h}_{1} \leq{ }_{h} \dot{h}_{2} \leq_{h} 1$ and the monotonicity property of any distance $\mathrm{d}(.,$.$) , we find that$ $\mathrm{d}\left(\left(\gamma_{1}^{1}, \ldots, \gamma_{m}^{1}\right), 1\right) \geq \mathrm{d}\left(\left(\gamma_{1}^{2}, \ldots, \gamma_{m}^{2}\right), 1\right)$ which easily implies that

$$
\operatorname{Sc}\left(\dot{h}_{1}\right)=1-\mathrm{d}\left(\left(\gamma_{1}^{1}, \ldots, \gamma_{m}^{1}\right), 1\right) \leq 1-\mathrm{d}\left(\left(\gamma_{1}^{2}, \ldots, \gamma_{m}^{2}\right), 1\right)=\operatorname{Sc}\left(\dot{h}_{2}\right)
$$

Property 2 (boundary conditions). Let $1=(1,1, \ldots, 1)$ and $0=(0,0, \ldots, 0)$ be One-SVEHFE and Zero-SVEHFE, respectively. Then, we conclude that the score function $\mathrm{Sc}$ (.) given by (22) satisfies

$$
S c(1)=1, \quad \text { and } S c(0)=0 .
$$

Proof. By keeping the axiom $0 \leq \mathrm{d}\left(\dot{h}_{1}, \dot{h}_{2}\right) \leq 1$ (for any SVEHFEs $\dot{h}_{1}$ and $\left.\dot{h}_{2}\right)$ in mind, the proof is evident.
Property 3 (idempotency). Let $\dot{h}=\left\{\left(\gamma_{1}, \ldots, \gamma_{m}\right)\right\}=$ $\{(\gamma, \ldots, \gamma)\}$. If $\mathrm{d}((\gamma, \ldots, \gamma), 1)=1-\gamma$, then the score function $S c($.$) given by (22) satisfies$

$$
S c(\dot{h})=\gamma .
$$

Proof. The proof is obvious.

Definition 3. If $S c($.$) stands for a score function of$ SVEHFEs, then

$$
D(S c(\dot{h}))=D\left(S c\left(\left\{\left(\gamma_{1}, \ldots, \gamma_{m}\right)\right\}\right)\right):=1-S c\left(\left\{\left(1-\gamma_{1}, \ldots, 1-\gamma_{m}\right)\right\}\right),
$$

which defines the dual form of $S c($.$) .$

$$
D(D(S c(\dot{h})))=S c(\dot{h}) .
$$

Property 4 (duality). The score function $S c($.$) given by (22)$ satisfies
Proof. Following from Definition 3, we get that 


$$
\begin{aligned}
D(D(S c(\dot{h}))) & =D\left(1-S c\left(\left\{\left(1-\gamma_{1}, \ldots, 1-\gamma_{m}\right)\right\}\right)\right) \\
& :=1-\left(1-S c\left(\left\{\left(1-\left(1-\gamma_{1}\right), \ldots, 1-\left(1-\gamma_{m}\right)\right)\right\}\right)\right)=S c\left(\left\{\left(\gamma_{1}, \ldots, \gamma_{m}\right)\right\}\right) .
\end{aligned}
$$

Property 5 (generalization). Let $\Theta:[0,1] \longrightarrow[0,1]$ be a strictly monotone decreasing real function and $\mathrm{d}(.$, .) be a distance measure between SVEHFEs. Then,

$$
S c_{\Theta}(\dot{h})=\Theta\left(\mathrm{d}\left(\left(\gamma_{1}, \ldots, \gamma_{m}\right), 1\right)\right),
$$

which defines a score function for SVEHFEs.

Proof. We show that $S c_{\Theta}$ (.) satisfies two fundamental properties, called above as monotonicity and boundary conditions.

Monotonicity property: from the fact that $\dot{h}_{1} \leq{ }_{h} \dot{h}_{2} \leq_{h} 1$ and the monotonicity property of any distance $\mathrm{d}(.,$.$) , we$ find that $\mathrm{d}\left(\left(\gamma_{1}^{1}, \ldots, \gamma_{m}^{1}\right), 1\right) \geq \mathrm{d}\left(\left(\gamma_{1}^{2}, \ldots, \gamma_{m}^{2}\right), 1\right)$. On the contrary, the latter inequality and the strictly monotone decreasing property of $\Theta$ give rise to

$$
\Theta\left(\mathrm{d}\left(\left(\gamma_{1}^{1}, \ldots, \gamma_{m}^{1}\right), 1\right)\right) \leq \Theta\left(\mathrm{d}\left(\left(\gamma_{1}^{2}, \ldots, \gamma_{m}^{2}\right), 1\right)\right),
$$

which implies that

$$
S c_{\Theta}\left(\dot{h}_{1}\right) \leq S c_{\Theta}\left(\dot{h}_{2}\right) .
$$

Boundary conditions' property: consider the One-SVEHFE $1=(1,1, \ldots, 1)$ and the Zero-SVEHFE $0=(0,0, \ldots, 0)$. Then, by keeping the axiom $0 \leq \mathrm{d}\left(\dot{h}_{1}, \dot{h}_{2}\right) \leq 1$ (for any SVEHFEs $\dot{h}_{1}$ and $\dot{h}_{2}$ ) in mind, we conclude easily that the score function $S c_{\Theta}$ (.) given by (30) satisfies

$$
S c_{\Theta}(1)=1, \quad \text { and } S c_{\Theta}(0)=0 .
$$

By the help of Property 5, we will be able to develop different formulas of score functions for SVEHFEs by taking into account different strictly monotone decreasing functions $\Theta:[0,1] \longrightarrow[0,1]$, for instance, $(1) \Theta_{1}(x)=1-x ;(2)$ $\Theta_{2}(x)=1-x / 1+x ; \quad$ (3) $\quad \Theta_{3}(x)=1-x e^{x-1}$; $\Theta_{4}(x)=1-x^{2}$.

From this property, the following SVEHFE-score functions can be established:

$$
\begin{aligned}
& S c_{\Theta_{1}}(\dot{h})=1-\mathrm{d}\left(\left(\gamma_{1}, \ldots, \gamma_{m}\right), 1\right) ; \\
& S c_{\Theta_{2}}(\dot{h})=\frac{1-\mathrm{d}\left(\left(\gamma_{1}, \ldots, \gamma_{m}\right), 1\right)}{1+\mathrm{d}\left(\left(\gamma_{1}, \ldots, \gamma_{m}\right), 1\right)} \\
& S c_{\Theta_{3}}(\dot{h})=1-\mathrm{d}\left(\left(\gamma_{1}, \ldots, \gamma_{m}\right), 1\right) e^{\mathrm{d}\left(\left(\gamma_{1}, \ldots, \gamma_{m}\right), 1\right)-1} ; \\
& S c_{\Theta_{4}}(\dot{h})=1-\mathrm{d}^{2}\left(\left(\gamma_{1}, \ldots, \gamma_{m}\right), 1\right) .
\end{aligned}
$$

\section{SVEHFS Score-Based Multiple Criteria Decision-Making}

This section provides three practical case studies to demonstrate that the proposed concept of SVEHFS is effective enough in the field of score-based optimization methods.
Briefly speaking, the proposed SVEHFS score-based decision-making procedure is composed of the following three stages: the unification process of PHFSs, the reduction process of PHFSs to SVEHFSs, and the selection procedure. The first two stages have been served in Sections 2 and 3. The last stage given in Section 4 describes the process of ranking alternatives in accordance with their values of score function and selecting the best one with the greatest value.

Now, in order for more systematically being understood, we give following Algorithm 3 (see Figure 4).

5.1. Case Study I. In this portion, we adopt an optimization problem which was originally solved in [33] by the use of a probabilistic linguistic term set- (PLTS-) based algorithm. Here, in order to have a better understanding of how the proposed SVEHFS- (or PHFS-) based algorithm behaves over the later-mentioned multiple criteria decision-making problem, we transform PLTS information to PHFS (or SVEHFS) data. This is done by the help of Theorem 1 in [34] in which the bijective transformation between PLTSs and PHFSs is explained.

A company needs to plan the development of large projects (strategy initiatives) for the next five years. To do this end, the company invites five experts to form the board of directors. Moreover, the company takes three possible projects $A_{i}(i=1,2$, and 3$)$ into consideration which should be evaluated based on their importance. These projects should be ranked in accordance with four criteria of the benefit type which are suggested by the balanced scorecard methodology as follows:

$$
\begin{aligned}
& C_{1}: \text { financial perspective } \\
& C_{2}: \text { the customer satisfaction } \\
& C_{3}: \text { internal business process perspective } \\
& C_{4}: \text { learning and growth perspective }
\end{aligned}
$$

Now, by adopting Algorithm 3 and the assumption that five experts apply the linguistic term set $S=\left\{s_{0}=\right.$ none, $s_{1}=$ verylow, $s_{2}=$ low, $s_{3}=$ medium, $s_{4}=$ high, $s_{5}=$ veryhigh, $s_{6}=$ perfect $\}$, we are able to evaluate the projects $A_{i}(i=$ 1,2 , and 3) by means of PLTSs in Step 1. The corresponding data is presented in Table 1.

To save more space, we only present the transformation form of the probabilistic linguistic decision matrix into that of PHFS as explained above. Consequently, the result will be that given in Table 2 .

Now, by the help of Step 2 of Algorithm 3, we are in a position to use the proposed unification process for the data of Table 2 and draw those results being summarized in Table 3.

In what follows, by the use of Step 3 of Algorithm 3, we will derive the corresponding SVEHFEs, as shown in Table 4.

If we now consider the weight vector of criteria $C_{i}(i=$ $1,2,3,4)$ in the form of $w=(0.138,0.304,0.416,0.142)$ 
Input: the probabilistic hesitant fuzzy decision matrix

Output: the ranking of alternatives and the best one

Step 1: build the probabilistic hesitant fuzzy decision matrix

Step 2: extract the unified form of PHFSs from the decision matrix

Step 3: reduced the probabilistic-unified PHFSs to so-called SVEHFSs

Step 4: find the best alternative(s) in accordance with their SVEHFS score values

Algorithm 3: Proposed SVEHFS score-based decision-making algorithm.

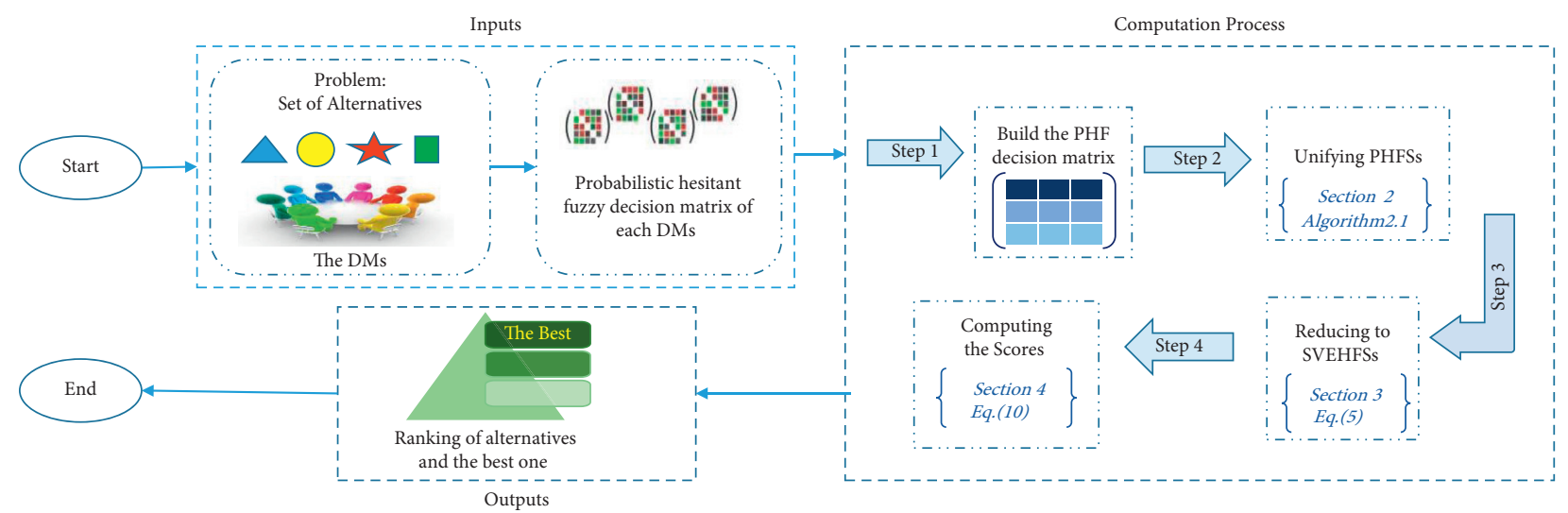

FIgURE 4: Proposed SVEHFS score-based decision-making algorithm.

Table 1: The probabilistic linguistic decision matrix.

\begin{tabular}{ccccc}
\hline & $C_{1}$ & $C_{2}$ & $C_{3}$ & $C_{4}$ \\
\hline$A_{1}$ & $\left\{s_{3}(0.4), s_{4}(0.6)\right\}$ & $\left\{s_{2}(0.2), s_{4}(0.8)\right\}$ & $\left\{s_{3}(0.2), s_{4}(0.8)\right\}$ & $\left\{s_{3}(0.4), s_{5}(0.6)\right\}$ \\
$A_{2}$ & $\left\{s_{3}(0.8), s_{5}(0.2)\right\}$ & $\left\{s_{2}(0.3), s_{3}(0.4), s_{4}(0.3)\right\}$ & $\left\{s_{1}(0.3), s_{2}(0.4), s_{3}(0.3)\right\}$ & $\left\{s_{3}(0.8), s_{4}(0.2)\right\}$ \\
$A_{3}$ & $\left\{s_{3}(0.6), s_{4}(0.4)\right\}$ & $\left\{s_{3}(0.6), s_{4}(0.2), s_{5}(0.2)\right\}$ & $\left\{s_{3}(0.4), s_{4}(0.2), s_{5}(0.4)\right\}$ & $\left\{s_{4}(0.7), s_{6}(0.3)\right\}$ \\
\hline
\end{tabular}

TABle 2: The probabilistic hesitant fuzzy decision matrix.

\begin{tabular}{ccccc}
\hline & $C_{1}$ & $C_{2}$ & $C_{3}$ & $C_{4}$ \\
\hline$A_{1}$ & $\{0.5(0.4), 0.67(0.6)\}$ & $\{0.33(0.2), 0.67(0.8)\}$ & $\{0.5(0.2), 0.67(0.8)\}$ & $\{0.5(0.4), 0.83(0.6)\}$ \\
$A_{2}$ & $\{0.5(0.8), 0.83(0.2)\}$ & $\{0.33(0.3), 0.5(0.4), 0.67(0.3)\}$ & $\{0.17(0.3), 0.33(0.4), 0.5(0.3)\}$ & $\{0.5(0.8), 0.67(0.2)\}$ \\
$A_{3}$ & $\{0.5(0.6), 0.67(0.4)\}$ & $\{0.5(0.6), 0.67(0.2), 0.83(0.2)\}$ & $\{0.5(0.4), 0.67(0.2), 0.83(0.4)\}$ & $\{0.67(0.7), 1(0.3)\}$ \\
\hline
\end{tabular}

together with $\lambda=1$ for distance measures $\mathrm{d}_{1}(.,),. \mathrm{d}_{2}(.,$.$) ,$ and $d_{3}(.,$.$) given, respectively, by (14)-(16); then, following$ Step 4 of Algorithm 3, the proposed SVEHFS-score function $S c$ (.) gives rise to the priorities of projects listed in Table 5. In addition to these results, the output of Pang et al.'s TOPSISbased and aggregation-based techniques [33] has been presented in Table 5.

Generally, the TOPSIS-based and aggregation-based techniques are chosen in accordance with the decision makers' need on one side, and on the other side, Pang et al.'s TOPSIS- and aggregation-based techniques [33] impose the extracondition of normalization by adding a number of artificial linguistic terms with "zero" probability. By imposing such artificial PLTS normalization process, the underlying optimization procedure will cause the computational process with more complexity. In contrast, the SVEHFS-score-based technique maintains the integrity and authenticity of decision information as far as possible, which results in much more reasonable decisions.

5.2. Case StudyII. In this part of contribution, we implement the proposed SVEHFS-score function for specifying the best Chinese hospital from a collection of considered hospitals. Such a problem was originally discussed by Song et al. [28], and then, it was more investigated by some other researchers 


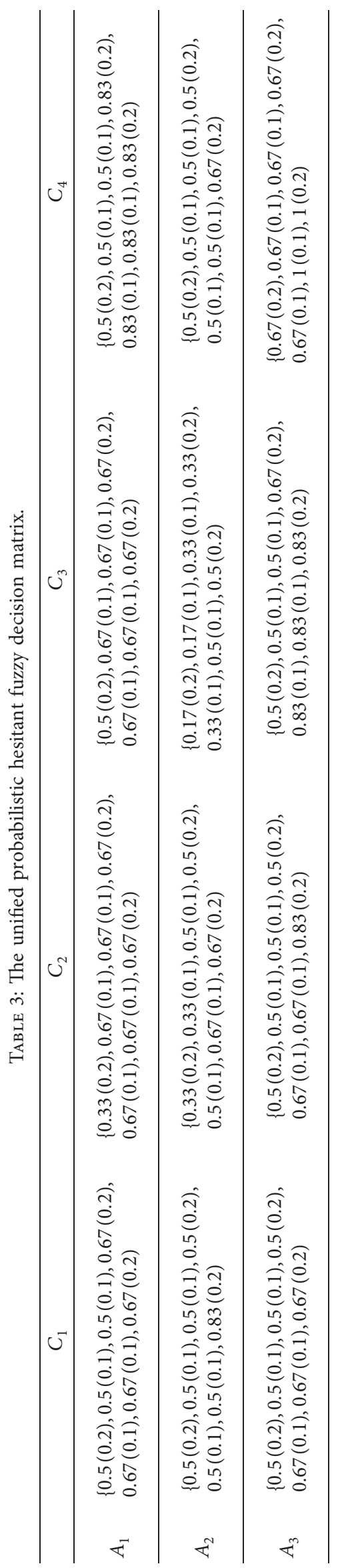




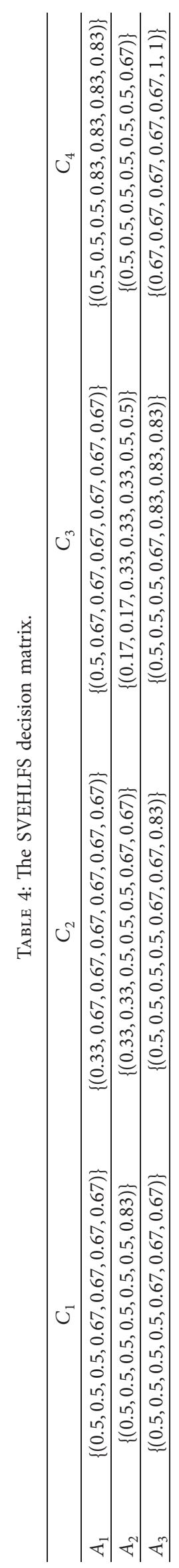


TABLE 5: Ranking results of the different techniques.

\begin{tabular}{lcc}
\hline & Ranking order & Optimal alternative \\
\hline Pang et al.'s TOPSIS-based technique [33] & $A_{1}>A_{3}>A_{2}$ & $A_{1}$ \\
Pang et al.'s aggregation-based technique [33] & $A_{1}>A_{3}>A_{2}$ & $A_{1}$ \\
The proposed SVEHFS-score function $S c_{d_{1}}$ & $A_{3}>A_{1}>A_{2}$ & $A_{3}$ \\
The proposed SVEHFS-score function $S c_{d_{2}}$ & $A_{3}>A_{1}>A_{2}$ & $A_{3}$ \\
The proposed SVEHFS-score function $S c_{d_{3}}$ & $A_{3}>A_{1}>A_{2}$ & $A_{3}$ \\
\hline
\end{tabular}

TABle 6: The probabilistic hesitant fuzzy decision matrix.

\begin{tabular}{lccc}
\hline & $\begin{array}{c}\text { Environment of health } \\
\text { service }\left(C_{1}\right)\end{array}$ & Treatment optimization $\left(C_{2}\right)$ & Social resource allocation $\left(C_{3}\right)$ \\
\hline West China Hospital $\left(A_{1}\right)$ & $\{0.5(0.4), 0.7(0.6)\}$ & $\{0.9(1)\}$ & $\{0.3(0.2), 0.5(0.8)\}$ \\
Huashan Hospital $\left(A_{2}\right)$ & $\{0.8(0.3), 0.9(0.7)\}$ & $\{0.5(1)\}$ & $\{0.8(0.4), 0.9(0.6)\}$ \\
Union Medical College Hospital $\left(A_{3}\right)$ & $\{0.5(1)\}$ & $\{0.7(0.5), 0.9(0.5)\}$ & $\{0.8(0.6), 0.9(0.4)\}$ \\
PLA General Hospital $\left(A_{4}\right)$ & $\{0.8(0.5), 0.9(0.5)\}$ & $\{0.3(0.5), 0.6(0.5)\}$ & $\{0.7(1)\}$ \\
\hline
\end{tabular}

including Zhang et al. [21] and Farhadinia and HerreraViedma [23].

The problem here is that we are seeking the best Chinese hospital with regards to the medical resource restriction and the old-age limitation of target population. In this regard, three criteria are mainly considered as $C_{1}$ : environment of health service, $C_{2}$ : treatment optimization, and $C_{3}$ : social resource allocation. The corresponding weight vector of criteria is supposed to be $w=(0.2,0.1,0.7)$. For this optimization problem, we evaluate four candidate hospitals including $A_{1}$ : West China Hospital of Sichuan University, $A_{2}$ : Huashan Hospital of Fudan University, $A_{3}$ : Union Medical College Hospital, and $A_{4}$ : Chinese PLA General Hospital. Since one option is not able to describe the influence factor, a number of experts are asked to express their preferences related to the abovementioned hospitals based on available criteria in the form of PHFSs.

Now, performing Step 1 of Algorithm 3 leads to constructing the following probabilistic hesitant fuzzy decision matrix (see Table 6).

Similar to what is discussed in Section 3 and applying Steps 2 and 3 of Algorithm 3, we will derive the corresponding unified PHFEs' and SVEHFEs' matrices, as shown in Tables 7 and 8, respectively.

Following Step 4 of Algorithm 3, if we now consider the weight vector of criteria $C_{i}(i=1,2,3)$ in the form of $w=$ $(0.2,0.1,0.7)$ together with $\lambda=1$ for distance measures $d_{1}(.,),. d_{2}(.,$.$) , and d_{3}(.,$.$) given, respectively, by (14)-(16),$ then the proposed SVEHFS-score function Sc (.) gives rise to the priority of Chinese hospitals listed in Tables 9 and 10. In addition to these results, the output of Zhang et al.'s [21], Song et al.'s [28], and Farhadinia and Xu [22] techniques have been also presented in Tables 9 and 10 .

As what can be observed from Tables 9 and 10, the most repeated alternative is $A_{2}$ which implies that the most appropriate hospital is the Huashan Hospital of Fudan University. This is exactly what we observe from the last three rows of Table 10 dedicated to the results of proposed SVEHFS-score functions.
Now, let us conclude the part of this section with some discussions on the pros and cons of proposed SVEHFS-score functions. The techniques of Zhang et al. [21] and Song et al. [28] are restricted directly to the normalization process of PHFSs, and Farhadinia and $\mathrm{Xu}$ [22] techniques are related to the multiplying and exponential deformation formulas of each pair of possible membership degree and its associated probability. This is while the proposed SVEHFS-score functions do not change the original form of PHFSs, and this can be seen as a pro. The other significant advantage of SVEHFS-based score functions over the other abovementioned techniques is its ease of use.

5.3. Case Study III. Because of competition and limitation of research funding in universities of China, a few outstanding research topics are annually supported. In order to select the best research topic several aspects including practicality, innovativeness, and feasibility are taken into consideration.

In March 2018, the business school of university A in China asked three instructors to submit their research topics for evaluating which one is more suitable for granting the university research funding. In this project, three professors $D M_{k}(k=1,2$, and 3$)$ are invited for evaluating the quality of the three research topics $A_{i}(i=1,2$, and 3$)$ in accordance with three criteria: $C_{j=1}$ : innovativeness, $C_{j=2}$ : practicality, and $C_{j=3}$ : feasibility. All the criteria are benefit types, and all the corresponding evaluations of three professors $D M_{k}(k=$ 1,2 , and 3 ) are represented in the form of PHFE-based decision matrices (see Tables 11-13).

By applying Steps 2 and 3 of Algorithm 3, the individual unified PHFEs are computed as the data given in Tables 14-16.

Following the process discussed by $\mathrm{Li}$ et al. [35], the decision makers' weights are obtained as

$$
\varpi_{k}=\frac{\sum_{j=1}^{n} \sum_{i=1}^{m-1} \sum_{g=i+1}^{m} \mathrm{~d}\left(\gamma_{i j}^{k}, \gamma_{g j}^{k}\right)}{\sum_{k=1}^{z} \sum_{j=1}^{n} \sum_{i=1}^{m-1} \sum_{g=i+1}^{m} \mathrm{~d}\left(\gamma_{i j}^{k}, \gamma_{g j}^{k}\right)}, \quad k=1,2, \text { and } 3,
$$




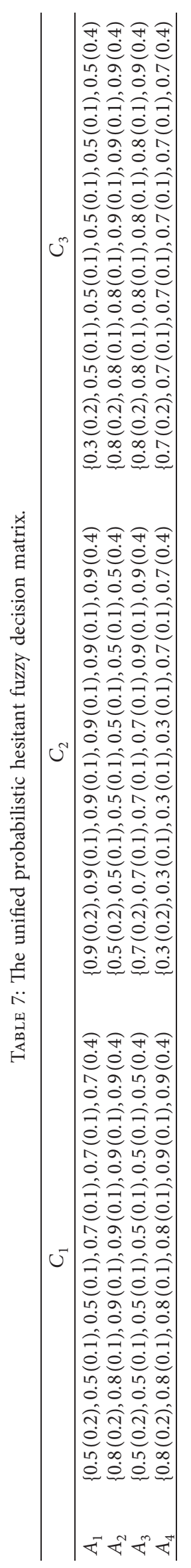


TABle 8: The SVEHFS decision matrix.

\begin{tabular}{llll}
\hline & $C_{1}$ & $C_{2}$ & $C_{3}$ \\
\hline$A_{1}$ & $\{(0.5,0.5,0.5,0.7,0.7,0.7)\}$ & $\{(0.9,0.9,0.9,0.9,0.9,0.9)\}$ & $\{(0.3,0.5,0.5,0.5,0.5,0.5)\}$ \\
$A_{2}$ & $\{(0.8,0.8,0.8,0.9,0.9,0.9)\}$ & $\{(0.5,0.5,0.5,0.5,0.5,0.5)\}$ & $\{(0.8,0.8,0.8,0.9,0.9,0.9)\}$ \\
$A_{3}$ & $\{(0.5,0.5,0.5,0.5,0.5,0.5)\}$ & $\{(0.7,0.7,0.7,0.7,0.9,0.9)\}$ & $\{(0.8,0.8,0.8,0.8,0.8,0.9)\}$ \\
$A_{4}$ & $\{(0.8,0.8,0.8,0.8,0.9,0.9)\}$ & $\{(0.3,0.3,0.3,0.3,0.7,0.7)\}$ & $\{(0.7,0.7,0.7,0.7,0.7,0.7)\}$ \\
\hline
\end{tabular}

TABle 9: Ranking results of Chinese hospitals.

\begin{tabular}{|c|c|c|c|}
\hline Score function & Score of hospitals & Ranking order & Optimal alternative \\
\hline $\bar{S}^{d_{1}}$ & $\begin{array}{lllll}0.8555 & 0.7884 & 0.8002 & 0.8186\end{array}$ & $A_{2}>A_{3}>A_{4}>A_{1}$ & $A_{2}$ \\
\hline$\underline{S}_{1}^{d_{1}}$ & $\begin{array}{llll}0.1268 & 0.0410 & 0.0546 & 0.0771\end{array}$ & $A_{2}>A_{3}>A_{4}>A_{1}$ & $A_{2}$ \\
\hline$\overline{\bar{S}}^{d_{2}}$ & $\begin{array}{llll}0.8618 & 0.7951 & 0.8007 & 0.8186\end{array}$ & $A_{2}>A_{3}>A_{4}>A_{1}$ & $A_{2}^{2}$ \\
\hline$\underline{S}_{1}^{d_{2}}$ & $\begin{array}{llll}0.1406 & 0.0432 & 0.0595 & 0.0774\end{array}$ & $A_{2}>A_{3}>A_{4}>A_{1}$ & $A_{2}$ \\
\hline$\overline{\bar{S}}^{d_{3}}$ & 0.96310 .90630 .83100 .8255 & $A_{4}>A_{3}>A_{2}>A_{1}$ & $A_{4}$ \\
\hline$\underline{S}_{d}^{d_{3}}$ & 0.20750 .05760 .08270 .0860 & $A_{2}>A_{3}>A_{4}>A_{1}$ & $A_{2}$ \\
\hline$\overline{\bar{S}}^{d_{4}}$ & 0.90930 .84730 .81560 .8220 & $A_{3}>A_{4}>A_{2}>A_{1}$ & $A_{3}$ \\
\hline$\underline{S}^{d_{4}}$ & 0.16710 .04930 .06860 .0815 & $A_{2}>A_{3}>A_{4}>A_{1}$ & $A_{2}$ \\
\hline$\overline{\bar{S}}_{A M}$ & 0.14450 .21160 .19980 .1814 & $A_{2}>A_{3}>A_{4}>A_{1}$ & $A_{2}$ \\
\hline$\underline{S}_{A M}^{A M}$ & $\begin{array}{llll}0.8732 & 0.9590 & 0.9454 & 0.9229\end{array}$ & $A_{2}>A_{3}>A_{4}>A_{1}$ & $A_{2}$ \\
\hline$\overline{\bar{S}}_{G M}^{A M}$ & 0.10540 .18710 .19770 .1814 & $A_{3}>A_{2}>A_{4}>A_{1}$ & $A_{3}$ \\
\hline$\underline{S}_{G M}^{G M}$ & $\begin{array}{llll}0.8711 & 0.9589 & 0.9451 & 0.9229\end{array}$ & $A_{2}>A_{3}>A_{4}>A_{1}$ & $A_{2}$ \\
\hline$\overline{\bar{S}}_{\operatorname{Min}}^{G M}$ & 0.03690 .09370 .16900 .1745 & $A_{4}>A_{3}>A_{2}>A_{1}$ & $A_{4}$ \\
\hline$\underline{S}_{M i n}^{\operatorname{Min}}$ & $\begin{array}{llll}0.7925 & 0.9424 & 0.9173 & 0.9140\end{array}$ & $A_{2}>A_{3}>A_{4}>A_{1}$ & $A_{2}$ \\
\hline$\overline{\bar{S}}_{\operatorname{Max}}^{\operatorname{Min}}$ & $0.2957 \quad 0.37040 .23280 .1880$ & $A_{2}>A_{1}>A_{3}>A_{4}$ & $A_{2}$ \\
\hline$\underline{\underline{S}}_{\operatorname{Max}}^{\operatorname{Max}}$ & 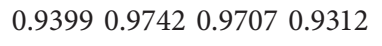 & $A_{2}>A_{3}>A_{1}>A_{4}$ & $A_{2}$ \\
\hline$\overline{\bar{S}}_{p}^{\operatorname{Max}}$ & 0.00010 .00120 .00150 .0011 & $A_{3}>A_{2}>A_{4}>A_{1}$ & $A_{3}$ \\
\hline$\underline{S}_{P}^{P}$ & 0.57580 .84550 .79790 .7255 & $A_{2}>A_{3}>A_{1}>A_{4}$ & $A_{2}$ \\
\hline$\overline{\bar{S}}_{B S}^{P}$ & 0.57790 .84640 .79920 .7257 & $A_{2}>A_{3}>A_{1}>A_{4}$ & $A_{2}$ \\
\hline$\underline{\underline{S}}_{B S}^{B S}$ & $\begin{array}{llll}1 & 1 & 1\end{array}$ & $A_{1}>A_{2}>A_{3}>A_{4}$ & $A_{1}$ \\
\hline$\overline{\bar{S}}_{F}^{B S}$ & 0.00070 .01120 .01120 .0069 & $A_{3}>A_{2}>A_{4}>A_{1}$ & $A_{3}$ \\
\hline$\underline{S}_{F}^{F}$ & 0.99931 .00001 .00000 .9998 & $A_{2}>A_{3}>A_{1}>A_{4}$ & $A_{2}$ \\
\hline
\end{tabular}

Table 10: Continues from Table 9.

\begin{tabular}{|c|c|c|}
\hline Technique & Ranking order & $\begin{array}{c}\text { Optimal } \\
\text { alternative }\end{array}$ \\
\hline Zhang et al.'s [21] & $A_{2}>A_{3}>A_{4}>A_{1}$ & $A_{2}$ \\
\hline Song et al.'s [28] & $A_{2}>{ }^{0.838} A_{3}>{ }^{0.819} A_{4}>{ }^{1} A_{1}$ & $A_{2}$ \\
\hline $\begin{array}{l}\text { Farhadinia and Xu's [22] first two step-based process multiplying deformation } \\
\text { formula: } \bar{S}_{1}\left(A_{1}\right)=0.144, \bar{S}_{1}\left(A_{2}\right)=0.2116, \bar{S}_{1}\left(A_{3}\right)=0.199 \text {, and } \bar{S}_{1}\left(A_{4}\right)=0.1814\end{array}$ & $A_{2}>A_{3}>A_{4}>A_{1}$ & $A_{2}$ \\
\hline $\begin{array}{l}\text { Exponential deformation formula: } \underline{S}_{1}\left(A_{1}\right)=0.873, \underline{S}_{1}\left(A_{2}\right)=0.9590 \text {, } \\
\underline{S}_{1}\left(A_{3}\right)=0.945 \text {, and } \underline{S}_{1}\left(A_{4}\right)=0.9229\end{array}$ & $A_{2}>A_{3}>A_{4}>A_{1}$ & $A_{2}$ \\
\hline $\begin{array}{l}\text { Farhadinia and Xu's [22] second two step-based process multiplying } \\
\text { deformation formula: } \bar{S}_{2}\left(A_{1}\right)=0.1445, \bar{S}_{2}\left(A_{2}\right)=0.2116, \bar{S}_{2}\left(A_{3}\right)=0.1998 \text {, and } \\
\bar{S}_{2}\left(A_{4}\right)=0.1814\end{array}$ & $A_{2}>A_{3}>A_{4}>A_{1}$ & $A_{2}$ \\
\hline $\begin{array}{l}\text { Exponential deformation formula: } \underline{S}_{2}\left(A_{1}\right)=0.8732, \underline{S}_{2}\left(A_{2}\right)=0.9590 \\
\underline{S}_{2}\left(A_{3}\right)=0.9454, \text { and } \underline{S}_{2}\left(A_{4}\right)=0.9229\end{array}$ & $A_{2}>A_{3}>A_{4}>A_{1}$ & $A_{2}$ \\
\hline $\begin{array}{l}\text { Farhadinia and Xu's [22] third two step-based process multiplying deformation } \\
\text { formula: } \bar{R}_{3}\left(A_{1}\right)=(0.1445,0.1376), \bar{R}_{3}\left(A_{2}\right)=(0.2116,0.1171) \\
\bar{R}_{3}\left(A_{3}\right)=(0.1998,0.0428), \bar{R}_{3}\left(A_{4}\right)=(0.1814,0.1824) \\
\end{array}$ & $\begin{array}{c}\bar{R}_{3}\left(A_{2}\right) \geq_{\text {lex }} \bar{R}_{3}\left(A_{3}\right) \geq_{\text {lex }} \\
\bar{R}_{3}\left(A_{4}\right) \geq_{\text {lex }} \bar{R}_{3}\left(A_{1}\right) A_{2}>A_{3}>A_{4}>A_{1}\end{array}$ & $A_{2}$ \\
\hline $\begin{array}{l}\text { Exponential deformation formula: } \underline{R}_{3}\left(A_{1}\right)=(0.8732,0.1499), \\
\underline{R}_{3}\left(A_{2}\right)=(0.9590,0.1114), \underline{R}_{3}\left(A_{3}\right)=(0.9454,0.0475), \text { and } \\
\underline{R}_{3}\left(A_{4}\right)=(0.9229,0.0430)\end{array}$ & $\begin{array}{c}\underline{R}_{3}\left(A_{2}\right) \geq_{\text {lex }} \underline{R}_{3}\left(A_{3}\right) \geq_{\text {lex }} \\
\underline{R}_{3}\left(A_{4}\right) \geq_{\text {lex }} \underline{R}_{3}\left(A_{1}\right) A_{2}>A_{3}>A_{4}>A_{1}\end{array}$ & $A_{2}$ \\
\hline $\begin{array}{l}\text { The proposed SVEHFS-score function } S c_{d_{1}}: S c_{d_{1}}\left(A_{1}\right)=0.5471 \text {, } \\
S c_{d_{1}}\left(A_{2}\right)=0.8735, S c_{d_{1}}\left(A_{3}\right)=0.7483 \text {, and } S c_{d_{1}}\left(A_{4}\right)=0.7000\end{array}$ & $A_{2}>A_{3}>A_{4}>A_{1}$ & $A_{2}$ \\
\hline $\begin{array}{l}\text { The proposed SVEHFS-score function } S c_{d_{2}} S c_{d_{2}}\left(A_{1}\right)=0.4310 \\
S c_{d_{2}}\left(A_{2}\right)=0.7970, S c_{d_{2}}\left(A_{3}\right)=0.7300 \text {, and } S c_{d_{2}}\left(A_{4}\right)=0.6800\end{array}$ & $A_{2}>A_{3}>A_{4}>A_{1}$ & $A_{2}$ \\
\hline $\begin{array}{l}\text { The proposed SVEHFS-score function } S c_{d_{2}} S c_{d_{3}}\left(A_{1}\right)=0.4890 \text {, } \\
S c_{d_{3}}\left(A_{2}\right)=0.8352, S c_{d_{3}}\left(A_{3}\right)=0.7391 \text {, and } S c_{d_{3}}\left(A_{4}\right)=0.6900\end{array}$ & $A_{2}>A_{3}>A_{4}>A_{1}$ & $A_{2}$ \\
\hline
\end{tabular}


TABLE 11: Evaluation information provided by $D M_{1}$.

\begin{tabular}{lccc}
\hline & $C_{1}$ & $C_{2}$ & $C_{3}$ \\
\hline$A_{1}$ & $\{0.3(0.3), 0.4(0.4), 0.5(0.3)\}$ & $\{0.4(0.3), 0.5(0.4), 0.6(0.3)\}$ & $\{0.2(1)\}$ \\
$A_{2}$ & $\{0.7(1)\}$ & $\{0.3(0.5), 0.4(0.5)\}$ & $\{0.8(0.5), 0.9(0.5)\}$ \\
$A_{3}$ & $\{0.6(0.5), 0.8(0.5)\}$ & $\{0.7(0.5), 0.9(0.5)\}$ & $\{0.3(0.5), 0.4(0.5)\}$ \\
\hline
\end{tabular}

TABLE 12: Evaluation information provided by $\mathrm{DM}_{2}$.

\begin{tabular}{cccc}
\hline & $C_{1}$ & $C_{2}$ & $C_{3}$ \\
\hline$A_{1}$ & $\{0.4(0.5), 0.5(0.5)\}$ & $\{0.6(1)\}$ & $\{0.5(0.3), 0.7(0.4), 0.8(0.3)\}$ \\
$A_{2}$ & $\{0.3(0.5), 0.4(0.5)\}$ & $\{0.4(0.3), 0.5(0.4), 0.6(0.3)\}$ & $\{0.6(0.5), 0.7(0.5)\}$ \\
$A_{3}$ & $\{0.5(0.5), 0.6(0.5)\}$ & $\{0.8(0.5), 0.9(0.5)\}$ & $\{0.6(1)\}$ \\
\hline
\end{tabular}

TABLE 13: Evaluation information provided by $\mathrm{DM}_{3}$.

\begin{tabular}{lccc}
\hline & $C_{1}$ & $C_{2}$ & $C_{3}$ \\
\hline$A_{1}$ & $\{0.1(0.5), 0.3(0.5)\}$ & $\{0.3(0.3), 0.4(0.4), 0.5(0.3)\}$ & $\{0.6(0.5), 0.7(0.5)\}$ \\
$A_{2}$ & $\{0.7(0.3), 0.8(0.4), 0.9(0.3)\}$ & $\{0.5(0.3), 0.6(0.4), 0.8(0.3)\}$ & $\{0.3(1)\}$ \\
$A_{3}$ & $\{0.4(0.5), 0.5(0.5)\}$ & $\{0.9(1)\}$ & $\{0.7(0.5), 0.8(0.5)\}$ \\
\hline
\end{tabular}

Table 14: The unified probabilistic hesitant fuzzy decision matrix for $D M_{1}$.

\begin{tabular}{llcc}
\hline & $C_{1}$ & $C_{2}$ & $C_{3}$ \\
\hline$A_{1}$ & $\{0.3(0.3), 0.4(0.2), 0.4(0.2), 0.5(0.3)\}$ & $\{0.4(0.3), 0.5(0.2), 0.5(0.2), 0.6(0.3)\}$ & $\{0.2(0.3), 0.2(0.2), 0.2(0.2), 0.2(0.3)\}$ \\
$A_{2}$ & $\{0.7(0.3), 0.7(0.2), 0.7(0.2), 0.7(0.3)\}$ & $\{0.3(0.3), 0.3(0.2), 0.4(0.2), 0.4(0.3)\}$ & $\{0.8(0.3), 0.8(0.2), 0.9(0.2), 0.9(0.3)\}$ \\
$A_{3}$ & $\{0.6(0.3), 0.6(0.2), 0.8(0.2), 0.8(0.3)\}$ & $\{0.7(0.3), 0.7(0.2), 0.9(0.2), 0.9(0.3)\}$ & $\{0.3(0.3), 0.3(0.2), 0.4(0.3), 0.4(0.3)\}$ \\
\hline
\end{tabular}

TABLE 15: The unified probabilistic hesitant fuzzy decision matrix for $D M_{2}$.

\begin{tabular}{cccc}
\hline & $C_{1}$ & $C_{2}$ & $C_{3}$ \\
\hline$A_{1}$ & $\{0.4(0.3), 0.4(0.2), 0.5(0.2), 0.5(0.3)\}$ & $\{0.6(0.3), 0.6(0.2), 0.6(0.2), 0.6(0.3)\}$ & $\{0.5(0.3), 0.7(0.2), 0.7(0.2), 0.8(0.3)\}$ \\
$A_{2}$ & $\{0.3(0.3), 0.3(0.2), 0.4(0.2), 0.4(0.3)\}$ & $\{0.4(0.3), 0.5(0.2), 0.5(0.2), 0.6(0.3)\}$ & $\{0.6(0.3), 0.6(0.2), 0.7(0.2), 0.7(0.3)\}$ \\
$A_{3}$ & $\{0.5(0.3), 0.5(0.2), 0.6(0.2), 0.6(0.3)\}$ & $\{0.8(0.3), 0.8(0.2), 0.9(0.2), 0.9(0.3)\}$ & $\{0.6(0.3), 0.6(0.2), 0.6(0.2), 0.6(0.3)\}$ \\
\hline
\end{tabular}

TABLE 16: The unified probabilistic hesitant fuzzy decision matrix for $\mathrm{DM}_{3}$.

\begin{tabular}{cccc}
\hline & $C_{1}$ & $C_{2}$ & $C_{3}$ \\
\hline$A_{1}$ & $\{0.1(0.3), 0.1(0.2), 0.3(0.2), 0.3(0.3)\}$ & $\{0.3(0.3), 0.4(0.2), 0.4(0.2), 0.5(0.3)\}$ & $\{0.6(0.3), 0.6(0.2), 0.7(0.2), 0.7(0.3)\}$ \\
$A_{2}$ & $\{0.7(0.3), 0.8(0.2), 0.8(0.2), 0.9(0.3)\}$ & $\{0.5(0.3), 0.6(0.2), 0.6(0.2), 0.8(0.3)\}$ & $\{0.3(0.3), 0.3(0.2), 0.3(0.2), 0.3(0.3)\}$ \\
$A_{3}$ & $\{0.4(0.3), 0.4(0.2), 0.5(0.2), 0.5(0.3)\}$ & $\{0.9(0.3), 0.9(0.2), 0.9(0.2), 0.9(0.3)\}$ & $\{0.7(0.3), 0.7(0.2), 0.8(0.2), 0.8(0.3)\}$ \\
\hline
\end{tabular}

TABle 17: The SVEHFS decision matrix for $D M_{1}$.

\begin{tabular}{lccc}
\hline & $C_{1}$ & $C_{2}$ & $C_{3}$ \\
\hline$A_{1}$ & $\{$ blue $(0.3,0.4,0.4,0.5)\}$ & $\{(0.4,0.5,0.5,0.6)\}$ & $\{(0.2,0.2,0.2,0.2)\}$ \\
$A_{2}$ & $\{0.7,0.7,0.7,0.7\}$ & $\{0.3,0.3,0.4,0.4\}$ & $\{0.8,0.8,0.9,0.9\}$ \\
$A_{3}$ & $\{0.6,0.6,0.8,0.8\}$ & $\{0.7,0.7,0.9,0.9\}$ & $\{0.3,0.3,0.4,0.4\}$ \\
\hline
\end{tabular}


TABLE 18: The SVEHFS decision matrix for $\mathrm{DM}_{2}$.

\begin{tabular}{cccc}
\hline & $C_{1}$ & $C_{2}$ & $C_{3}$ \\
\hline$A_{1}$ & $\{0.4,0.4,0.5,0.5\}$ & $\{0.6,0.6,0.6,0.6\}$ & $\{0.5,0.7,0.7,0.8\}$ \\
$A_{2}$ & $\{0.3,0.3,0.4,0.4\}$ & $\{0.4,0.5,0.5,0.6\}$ & $\{0.6,0.6,0.7,0.7\}$ \\
$A_{3}$ & $\{0.5,0.5,0.6,0.6\}$ & $\{0.8,0.8,0.9,0.9\}$ & $\{0.6,0.6,0.6,0.6\}$ \\
\hline
\end{tabular}

TABle 19: The SVEHFS decision matrix for $\mathrm{DM}_{3}$.

\begin{tabular}{lccc}
\hline & $C_{1}$ & $C_{2}$ & $C_{3}$ \\
\hline$A_{1}$ & $\{0.1,0.1,0.3,0.3\}$ & $\{0.3,0.4,0.4,0.5\}$ & $\{0.6,0.6,0.7,0.7\}$ \\
$A_{2}$ & $\{0.7,0.8,0.8,0.9\}$ & $\{0.5,0.6,0.6,0.8\}$ & $\{0.3,0.3,0.3,0.3\}$ \\
$A_{3}$ & $\{0.4,0.4,0.5,0.5\}$ & $\{0.9,0.9,0.9,0.9\}$ & $\{0.7,0.7,0.8,0.8\}$ \\
\hline
\end{tabular}

TABle 20: The collective SVEHFS decision matrix.

\begin{tabular}{cccc}
\hline & $C_{1}$ & $C_{2}$ & $C_{3}$ \\
\hline$A_{1}$ & $\{(0.2537,0.2934,0.3891,0.4273)\}$ & $\{0.4212,0.4901,0.4901,0.5623\}$ & $\{0.4602,0.5230,0.5753,0.6150\}$ \\
$A_{2}$ & $\{0.6317,0.6873,0.6987,0.7722\}$ & $\{0.4112,0.4851,0.5125,0.6508\}$ & $\{0.6079,0.6079,0.7140,0.7140\}$ \\
$A_{3}$ & $\{0.5028,0.5028,0.6577,0.6577\}$ & $\{0.8254,0.8254,0.9000,0.9000\}$ & $\{0.5657,0.5657,0.6508,0.6508\}$ \\
\hline
\end{tabular}

in which $\mathrm{d}(.,$.$) is a distance measure.$

If we implement distance measures $\mathrm{d}_{1}(.,),. \mathrm{d}_{2}(.,$.$) , and$ $\mathrm{d}_{3}(.,$.$) provided, respectively, by (14)-(16) with \lambda=1$, then the weight vectors will be

$$
\begin{aligned}
& \omega_{d_{1}}=\left(\Phi_{1}, \omega_{2}, \omega_{3}\right)=(0.3545,0.2421,0.4034) \text {, } \\
& \varpi_{d_{2}}=\left(\varpi_{1}, \varpi_{2}, \varpi_{3}\right)=(0.3864,0.1932,0.4204) \text {, } \\
& \omega_{d_{3}}=\left(\omega_{1}, \omega_{2}, \omega_{3}\right)=(0.3927,0.1859,0.4215) \text {. }
\end{aligned}
$$

To save more space for convenient storage, we only list the subsequent results for $\omega_{d_{1}}$.

Now, if we aggregate the individual SVEHFS matrices given in Tables $17-19$ by the help of the following rule

$$
\dot{h}=\oplus_{k=1}^{3}\left(\varpi_{k} \times \dot{h}_{k}\right)=\left\{\left(1-\prod_{k=1}^{3}\left(1-\dot{\hbar}_{k}\right)^{\Phi_{k}}\right)^{(1)}, \ldots,\left(1-\prod_{k=1}^{3}\left(1-\dot{\hbar}_{k}\right)^{\Phi_{k}}\right)^{(m)}\right\}
$$

in which $\omega_{k}$ stands for the weight of the decision makers $D M_{k}$ $(k=1,2$, and 3$)$ and the notation $(j)$ (for $j=1, \ldots, m) \mathrm{de}-$ notes the $j$ th element of collective SVEHFS, then the collective SVEHFS matrices can be derived in the form of Table 20.

Table 21 shows the comparison outcomes of different techniques. The ranking results obtained by the techniques of Xu et al. [36, 37] and Li et al. [35] are identical to those of proposed SVEHFS-score techniques. Such identical ranking results are possibly related to the same steps of processing which are performed using the latter-mentioned techniques. Briefly speaking, the common steps of these techniques are the integration of evaluation information given by the decision makers, the calculation of score value of the collective evaluation information, and the comparison of alternatives by the help of their score values. The outcomes of such identical steps are seen in identical ranking results.
But, the result of classical ORESTE technique [38] is quite different from that of other abovementioned techniques. Such a different ranking result arises from the twostage integrating ranking process. The initial stage calculates utility values for determining the weak ranking of alternatives. Then, the subsequent stage will derive the preference, indifference, and incomparability relations with conflict analysis. Finally, the strong ranking of alternatives is extracted. However, due to such complicated two-stage procedure, the best alternative obtained from classical ORESTE technique does not appear convincing enough.

In summary, the comparison with other considered techniques suggests that the proposed SVEHFS-score techniques have superior performance and also less computational complexity. 
TABLE 21: Ranking results of the different techniques.

\begin{tabular}{|c|c|c|c|c|c|}
\hline Technique & \multicolumn{3}{|c|}{ Score of alternatives } & Ranking order & Optimal alternative \\
\hline PHFWA-based technique of $\mathrm{Xu}$ et al. [36] & 0.4584 & 0.5990 & 0.7245 & $A_{1}<A_{2}<A_{3}$ & $A_{3}$ \\
\hline PHFWG-based technique of Xu et al. [36] & 0.4117 & 0.5284 & 0.6909 & $A_{1}<A_{2}<A_{3}$ & $A_{3}$ \\
\hline $\mathrm{Xu}$ et al. [37] & 0.2804 & 0.3653 & 0.7974 & $A_{1}<A_{2}<A_{3}$ & $A_{3}$ \\
\hline Classical ORESTE [38] & 4.8172 & 7.1306 & 6.7856 & $A_{2}<A_{3}<A_{1}$ & $A_{1}$ \\
\hline Li et al. [35] & 0.2563 & 0.1933 & 0.1212 & $A_{1}<A_{2}<A_{3}$ & $A_{3}$ \\
\hline The proposed SVEHFS-score function $S c_{d_{1}}$ & 0.4583 & 0.6244 & 0.6837 & $A_{1}<A_{2}<A_{3}$ & $A_{3}$ \\
\hline The proposed SVEHFS-score function $S c_{d_{2}}$ & 0.3784 & 0.5503 & 0.6313 & $A_{1}<A_{2}<A_{3}$ & $A_{3}$ \\
\hline The proposed SVEHFS-score function $S c_{d_{3}}$ & 0.4183 & 0.5873 & 0.6575 & $A_{1}<A_{2}<A_{3}$ & $A_{3}$ \\
\hline
\end{tabular}

\section{Conclusion}

Adopting a probability splitting algorithm for deriving an efficient probabilistic-unification process of PHFSs, we developed a class of score functions for SVEHFSs which are novel deformation of PHFSs. As we demonstrated here, the concept of SVEHFS belongs to a less dimensional space compared to that of PHFSs. Furthermore, we indicated that the proposed SVEHFS-based score functions satisfy a number of interesting properties. It may be of interest to mention that the proposed SVEHFS-based score functions are able to be more generalized to a wider class. Lastly, three case studies were prepared to illustrate the applicability and efficiency of proposed SVEHFS-based score functions compared to other existing PHFS-based techniques. In contrast to the other existing techniques for PHFSs, the SVEHFS-based score functions are associated with less complexity and computation requirements.

In future work, we will work towards opportunities to investigate the meaning and essence of SVEHFS-based score functions in the MCDM under probabilistic hesitant fuzzy setting.

\section{Data Availability}

No data were used to support this study.

\section{Conflicts of Interest}

The authors declare that they have no conflicts of interest.

\section{References}

[1] V. Torra, "Hesitant fuzzy sets," International Journal of Intelligent Systems, vol. 25, pp. 529-539, 2010.

[2] B. Farhadinia, "A multiple criteria decision making model with entropy weight in an interval-transformed hesitant fuzzy environment," Cognitive Computation, vol. 9, no. 4, pp. 513-525, 2017.

[3] M. Xia and Z. Xu, "Hesitant fuzzy information aggregation in decision making," International Journal of Approximate Reasoning, vol. 52, no. 3, pp. 395-407, 2011.

[4] R. M. Rodriguez, L. Martinez, and F. Herrera, "Hesitant fuzzy linguistic term sets for decision making," IEEE Transactions on Fuzzy Systems, vol. 20, no. 1, pp. 109-119, 2012.

[5] B. Farhadinia and Z. Xu, "Novel hesitant fuzzy linguistic entropy and cross-entropy measures in multiple criteria decision making," Applied Intelligence, vol. 48, no. 11, pp. 3915-3927, 2018.
[6] G. Qian, H. Wang, and X. Feng, "Generalized hesitant fuzzy sets and their application in decision support system," Knowledge-Based Systems, vol. 37, pp. 357-365, 2013.

[7] B. Zhu and Z. Xu, "Extended hesitant fuzzy sets," Technological and Economic Development of Economy, vol. 22, no. 1, pp. 100-121, 2016.

[8] B. Farhadinia, "Similarity-based multi-criteria decision making technique of pythagorean fuzzy sets," Artificial Intelligence Review, 2021.

[9] B. Zhu and Z. Xu, "Probability-hesitant fuzzy sets and the representation of preference relations," Technological and Economic Development of Economy, vol. 24, no. 3, pp. 10291040, 2018.

[10] S. Das, D. Malakar, S. Kar, and T. Pal, "Correlation measure of hesitant fuzzy soft sets and their application in decision making," Neural Computing \& Applications, vol. 31, no. 4, pp. 1023-1039, 2019.

[11] A. De, S. Das, and S. Kar, "Multiple attribute decision making based on probabilistic interval-valued intuitionistic hesitant fuzzy set and extended TOPSIS method," Journal of Intelligent and Fuzzy Systems, vol. 37, no. 4, pp. 5229-5248, 2019.

[12] R. Krishankumar, K. S. Ravichandran, S. Kar, P. Gupta, and M. K. Mehlawat, "Interval-valued probabilistic hesitant fuzzy set for multi-criteria group decision-making," Soft Computing, vol. 23, no. 21, pp. 10853-10879, 2019.

[13] S. Dozic, T. Lutovac, and M. Kalic, "Fuzzy AHP approach to passenger aircraft type selection," Journal of Air Transport Management, vol. 68, pp. 165-175, 2018.

[14] D.-H. Peng, C.-Y. Gao, and Z.-F. Gao, "Generalized hesitant fuzzy synergetic weighted distance measures and their application to multiple criteria decision-making," Applied Mathematical Modelling, vol. 37, no. 8, pp. 5837-5850, 2013.

[15] Z. Zhang and C. Wu, "Weighted hesitant fuzzy sets and their application to multi-criteria decision making," British Journal of Mathematics \& Computer Science, vol. 4, no. 8, pp. 1091-1123, 2014.

[16] J. Li and J.-Q. Wang, "An extended QUALIFLEX method under probability hesitant fuzzy environment for selecting green suppliers," International Journal of Fuzzy Systems, vol. 19, no. 6, pp. 1866-1879, 2017.

[17] L. Yue, M. Sun, and Z. Shao, "The probabilistic hesitant fuzzy weighted average operators and their application in strategic decision making," Journal of Information and Computational Science, vol. 10, no. 12, pp. 3841-3848, 2013.

[18] S. Zeng, J. M. Merigó, and W. Su, “The uncertain probabilistic OWA distance operator and its application in group decision making," Applied Mathematical Modelling, vol. 37, no. 9, pp. 6266-6275, 2013.

[19] Q. Ding, Y.-M. Wang, and M. Goh, "TODIM dynamic emergency decision-making method based on hybrid weighted distance under probabilistic hesitant fuzzy 
information," International Journal of Fuzzy Systems, vol. 23, no. 2, pp. 474-491, 2021.

[20] B. Farhadinia, "An innovative unification process for probabilistic hesitant fuzzy elements and its application to decision making," Fuzzy Optimization and Decision Making, 2021.

[21] S. Zhang, Z. Xu, and Y. He, "Operations and integrations of probabilistic hesitant fuzzy information in decision making," Information Fusion, vol. 38, pp. 1-11, 2017.

[22] B. Farhadinia and E. Herrera-Viedma, "Sorting of decisionmaking methods based on their outcomes using dominancevector hesitant fuzzy-based distance," Soft Computing, vol. 23, no. 4, pp. 1109-1121, 2019.

[23] B. Farhadinia and E. Herrera-Viedma, "A modification of probabilistic hesitant fuzzy sets and its application to multiple criteria decision making," Iranian Journal of Fuzzy Systems, 2020.

[24] J. Li and Z.-X. Wang, "Multi-attribute decision making based on prioritized operators under probabilistic hesitant fuzzy environments," Soft Computing, vol. 23, no. 11, pp. 3853-3868, 2019.

[25] X. Wu, H. Liao, Z. Xu, A. Hafezalkotob, and F. Herrera, "Probabilistic linguistic MULTIMOORA: a multicriteria decision making method based on the probabilistic linguistic expectation function and the improved Borda rule," IEEE Transactions on Fuzzy Systems, vol. 26, no. 6, pp. 3688-3702, 2018.

[26] M. Lin, Q. Zhan, and Z. Xu, "Decision making with probabilistic hesitant fuzzy information based on multiplicative consistency," International Journal of Intelligent Systems, vol. 35, no. 8, pp. 1233-1261, 2020.

[27] F. Jiang and Q. Ma, "Multi-attribute group decision making under probabilistic hesitant fuzzy environment with application to evaluate the transformation efficiency," Applied Intelligence, vol. 48, no. 4, pp. 953-965, 2018.

[28] C. Song, Z. Xu, and H. Zhao, "A novel comparison of probabilistic hesitant fuzzy elements in multi-criteria decision making," Symmetry, vol. 10, no. 5, p. 177, 2018.

[29] R. Krishankumar, K. S. Ravichandran, P. Liu, S. Kar, and A. H. Gandomi, "A decision framework under probabilistic hesitant fuzzy environment with probability estimation for multi-criteria decision making, Neural," Computer Applications, vol. 33, pp. 8417-8433, 2021.

[30] J. Wu, S. Zhang, Z. Wang, and H. Garg, "Extended cumulative residual entropy for emergency group decision-making under probabilistic hesitant fuzzy environment," International Journal of Fuzzy Systems, vol. 87, 2021.

[31] B. Farhadinia and $\mathrm{Z}$. Xu, "Developing the comparison techniques of probabilistic hesitant fuzzy elements in multiple criteria decision making," Soft Computing, vol. 25, no. 1, pp. 331-342, 2021.

[32] B. Zhu, Decision Method for Research and Application Based on Preference Relation, Southeast University, Nanjing, China, 2014.

[33] Q. Pang, H. Wang, and Z. Xu, "Probabilistic linguistic term sets in multi-attribute group decision making," Information Sciences, vol. 369, pp. 128-143, 2016.

[34] B. Farhadinia and Z. Xu, "Ordered weighted hesitant fuzzy information fusion-based approach to multiple attribute decision making with probabilistic linguistic term sets," Fundamenta Informaticae, vol. 159, no. 4, pp. 361-383, 2018.

[35] J. Li, Q. Chen, L. Niu, and Z. Wang, "An ORESTE approach for multi-criteria decision-making with probabilistic hesitant fuzzy information," International Journal of Machine Learning and Cybernetics, vol. 11, 2020.
[36] Z. Xu and W. Zhou, "Consensus building with a group of decision makers under the hesitant probabilistic fuzzy environment," Fuzzy Optimization and Decision Making, vol. 16, no. 4, pp. 481-503, 2017.

[37] Z. Xu and X. Zhang, "Hesitant fuzzy multi-attribute decision making based on TOPSIS with incomplete weight information," Knowledge-Based Systems, vol. 52, pp. 53-64, 2013.

[38] H. Pastijn and J. Leysen, "Constructing an outranking relation with ORESTE," Models and Methods in Multiple Criteria Decision Making, vol. 12, pp. 1255-1268, 1989. 